\title{
Epistemic logic meets epistemic game theory: a comparison between multi-agent Kripke models and type spaces
}

\author{
Paolo Galeazzi $^{1}$ • Emiliano Lorini ${ }^{2}$
}

Received: 28 August 2014 / Accepted: 18 July 2015 / Published online: 1 August 2015

(C) The Author(s) 2015. This article is published with open access at Springerlink.com

\begin{abstract}
In the literature there are at least two main formal structures to deal with situations of interactive epistemology: Kripke models and type spaces. As shown in many papers (see Aumann and Brandenburger in Econometrica 36:1161-1180, 1995; Baltag et al. in Synthese 169:301-333, 2009; Battigalli and Bonanno in Res Econ 53(2):149-225, 1999; Battigalli and Siniscalchi in J Econ Theory 106:356-391, 2002; Klein and Pacuit in Stud Log 102:297-319, 2014; Lorini in J Philos Log 42(6):863904, 2013), both these frameworks can be used to express epistemic conditions for solution concepts in game theory. The main result of this paper is a formal comparison between the two and a statement of semantic equivalence with respect to two different logical systems: a doxastic logic for belief and an epistemic-doxastic logic for belief and knowledge. Moreover, a sound and complete axiomatization of these logics with respect to the two equivalent Kripke semantics and type spaces semantics is provided. Finally, a probabilistic extension of the result is also presented. A further result of the paper is a study of the relationship between the epistemic-doxastic logic for belief and knowledge and the logic STIT (the logic of "seeing to it that") by Belnap and colleagues (Facing the future: agents and choices in our indeterminist world, 2001).
\end{abstract}

Keywords Epistemic game theory · Epistemic logic Type space

Interactive epistemology $\cdot$ STIT logic

\footnotetext{
$凶 \quad$ Paolo Galeazzi pagale87@gmail.com

Emiliano Lorini

Emiliano.Lorini@irit.fr

1 ILLC, University of Amsterdam, Amsterdam, The Netherlands

2 IRIT-CNRS, Toulouse University, Toulouse, France
} 


\section{Introduction}

In recent years many game theorists have focused on the epistemic part of playing a game, taking explicitly into account the knowledge and beliefs of the players involved in strategic interactions. The goal of the epistemic approach to game theory is to study what are the players' epistemic conditions that lead to solution concepts. Indeed, there are few games (e.g., the Prisoners' Dilemma) that do not need any strategic reasoning about the others for a rational player to choose an action. In most of the situations instead players have to take into consideration what they think about the others' actions and beliefs in order to choose an action. Furthermore, we need to consider what a player thinks about the others in order to assess her rationality. Strategic thinking comes out when the players reason about the others' actions, knowledge and beliefs, and epistemic game theory makes it explicit.

In the literature there are at least two main formal structures to deal with situations of interactive epistemology: Kripke models, mainly used in logic and computer science Fagin et al. (1995), and type spaces, more common in economics and game theory (Harsanyi 1967-1968). As shown in many papers, both these two frameworks can be used to express epistemic conditions for solution concepts. For instance, in Aumann and Brandenburger (1995) and Battigalli and Siniscalchi (2002) epistemic conditions for Nash equilibrium and extensive form rationalizability are shown by means of type spaces, whereas in Baltag et al. (2009) and Lorini (2013) epistemic conditions for backward induction and iterated weak dominance are expressed making use of Kripke models. The issue we try to address here is to formally study the relationship between these two structures, with a view to a possible broader communication and closer interaction between the two communities, epistemic logic and epistemic game theory.

Quite recently some steps have already been attempted towards this aim (Battigalli and Bonanno (1999), Heifetz and Mongin (2001), Klein and Pacuit (2014), Zvesper (2010)). In Heifetz and Mongin (2001) the authors are able to identify a logical system sound and complete with respect to the class of type spaces based on some modifications of Aumann's system (Aumann 1995). Both these logical systems are probabilistic, in the sense that they are expressed in a language with probabilistic operators. Zvesper's work instead starts from a qualitative version of type spaces, that he names type-space models and shows that there is an isomorphism with Kripkean state-space models.

The present work is connected with both these approaches. In Sect. 2, we start by defining a qualitative multi-agent epistemic language with belief operators. Then, we show firstly how to interpret it on a specific class of qualitative Kripke models, that we call doxastic game models, and later how to interpret the same language on probabilistic type spaces. Finally, we prove that the two frameworks are semantically equivalent with respect to the language.

In Sect. 3, we extend the language by introducing knowledge operators, in order to express two different epistemic attitudes in our frameworks. We show how to interpret the extended language on type spaces and subsequently we define the corresponding class of epistemic-doxastic game models. In the end we prove the semantic equiva- 
lence between the two semantics with respect to the extended language. Consequently, doxastic game models and epistemic-doxastic game models represent the qualitative Kripkean counterpart of type spaces.

Section 4 is devoted to the axiomatizations of the logic of belief and of the logic of belief and knowledge interpreted over doxastic game models and epistemic-doxastic game models, respectively. Given the results on the semantic side, these two logics are sound and complete with respect to type spaces too.

Section 5 presents a study of the relationship between such a logic of belief and knowledge and the logic STIT (the logic of "seeing to it that") by Belnap and colleagues (2001). The point of this study consists in building a connection between the area of epistemic logics and the area of logics of action.

We conclude in Sect. 6 by extending the analysis about the equivalence between Kripke-style semantics and type space semantics for epistemic modal languages to a modal language with probabilistic beliefs. The significance of this section consists in showing that the equivalence between the two kinds of semantics is preserved when moving from a qualitative representation of epistemic attitudes to a quantitative one based on probabilities.

\section{Models with belief}

In this section we will introduce the epistemic structures under consideration: doxastic Kripke models and type spaces. As usual in logic, we start out by introducing a logical language to talk about the epistemic interactive situation of the players in a game, and in the end we interpret it on both doxastic models and type spaces.

\subsection{Language}

Let us be given a finite simultaneous-move game $\mathbf{G}=\left\langle N,\left(A_{i}, \pi_{i}\right)_{i \in N}\right\rangle$, with $N$ the set of players, and $\forall i \in N$ a finite action set $A_{i}$ and a payoff function $\pi_{i}$. We want to endow ourselves with a logical language to talk about the epistemic situation of the players. We define the language $\mathscr{L}_{D G L}(\mathbf{G})$ for the doxastic game logic (DGL) as generated by the following grammar:

$$
\varphi::=p l\left(a_{i}\right)|\neg \varphi| \varphi \wedge \varphi \mid B_{i} \varphi
$$

where $a_{i} \in A_{i}$ and $i \in N$. The other boolean operators $\vee, \perp, \top, \rightarrow$ and $\leftrightarrow$ are defined in the standard way. The language $\mathscr{L}_{D G L}(\mathbf{G})$ is a doxastic language with a belief operator $B_{i}$ for each player $i$. Notice that the language $\mathscr{L}_{D G L}(\mathbf{G})$, and consequently the logic DGL, is parametrized by the game $\mathbf{G}$ that we are taking into consideration. It is the game $\mathbf{G}$ that gives us the primitives $p l\left(a_{i}\right)$ of our language $\mathscr{L}_{D G L}(\mathbf{G})$ : for each $a_{i} \in A_{i}$ we have one primitive $p l\left(a_{i}\right)$, read as "player $i$ is playing her action $a_{i}$ ". The doxastic formula $B_{i} \varphi$ has to be read "player $i$ believes that $\varphi$ is true". Let us define the sets $\Phi_{i}:=\left\{p l\left(a_{i}\right): a_{i} \in A_{i}\right\}$ and $\Phi:=\bigcup_{i \in N} \Phi_{i}$. Moreover, let us abbreviate $\widehat{B}_{i} \varphi:=\neg B_{i} \neg \varphi$. 


\subsection{Semantics}

\subsubsection{Doxastic models}

In the epistemic logic literature it is standard to express the semantics of doxastic languages by means of structures called doxastic models. Doxastic models are a specific type of Kripke models used in modal logic (Blackburn et al. 2001).

Definition 1 (Doxastic model) A doxastic model is a tuple $M=\left\langle W, \rightarrow_{1}, \ldots, \rightarrow_{n}, v\right\rangle$ where:

- $W$ is a countable set of possible worlds;

$-v: W \longrightarrow \mathscr{P}(\Phi)$ is the valuation function for the set $\Phi$ of atomic facts defined in Sect. 2.1 (with $\mathscr{P}(\Phi)$ the power set of $\Phi$ );

- $\rightarrow{ }_{i} \subseteq W \times W$ is the belief relation of player $i$ that satisfies the following conditions:

- seriality: $\forall w \exists w^{\prime}$ such that $w \rightarrow_{i} w^{\prime}$;

- transitivity: $\forall u, w, z: u \rightarrow_{i} w$ and $w \rightarrow_{i} z$ implies $u \rightarrow_{i} z$;

- Euclideaness: $\forall u, w, z: w \rightarrow_{i} u$ and $w \rightarrow_{i} z$ implies $u \rightarrow_{i} z$.

Let us define the belief set of player $i$ at world $w$ as follows: $\rightarrow_{i}(w):=\left\{w^{\prime}: w \rightarrow_{i} w^{\prime}\right\}$.

Before interpreting $\mathscr{L}_{D G L}(\mathbf{G})$ over doxastic models we are going to identify a subclass of doxastic models, that we call doxastic game models DGM for game G. A similar notion of doxastic game model is defined by Lorini and Schwarzentruber (2010).

Definition 2 (Doxastic game model) A doxastic game model for the game $\mathbf{G}$ is a doxastic model $M=\left\langle W, \rightarrow_{1}, \ldots, \rightarrow_{n}, v\right\rangle$ satisfying the following conditions:

- Adequate valuation condition (AVC):

$$
\forall i \in N, \forall w \in W, v_{i}(w) \text { is a singleton, }
$$

where $v_{i}(w)$ is the restriction of $v(w)$ to $\Phi_{i}$, i.e., $v_{i}(w)=v(w) \cap \Phi_{i}$;

- Ex interim condition (ExIC):

$$
\begin{aligned}
& \forall i \in N, \forall w, w^{\prime} \in W, \forall a_{i} \in A_{i}, \text { if } w \rightarrow_{i} w^{\prime} \text { and } p l\left(a_{i}\right) \in \\
& v(w) \text { then } \operatorname{pl}\left(a_{i}\right) \in v\left(w^{\prime}\right) .
\end{aligned}
$$

AVC simply says that the valuation function assigns one and only one action to each player at each world, since we do not want to have worlds in which a player can play two different actions at the same time. ExIC means that if a player plays an action, then she believes to play that action. For this reason we call it ex interim condition: it describes a stage in the game where the players have already chosen their own actions, and they might be uncertain only about the others' actions.

Doxastic game models of the form $M=\left\langle W, \rightarrow_{1}, \ldots, \rightarrow_{n}, v\right\rangle$ can be used to provide a semantics for the language $\mathscr{L}_{D G L}(\mathbf{G})$. The following are the truth conditions of formulas in the language $\mathscr{L}_{D G L}(\mathbf{G})$ relative to doxastic game models, where $M, w \vDash$ $\varphi$ means that formula $\varphi$ is true at world $w$ in the model $M$ : 


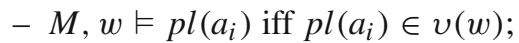

$-M, w \vDash \neg \varphi$ iff $M, w \not \models \varphi$;

$-M, w \vDash \varphi \wedge \psi$ iff $M, w \vDash \varphi$ and $M, w \vDash \psi$;

$-M, w \vDash B_{i} \varphi$ iff $\forall w^{\prime} \in W$, if $w \rightarrow_{i} w^{\prime}$ then $M, w^{\prime} \vDash \varphi$.

As usual we say that a formula $\varphi$ is true in a model $M=\left\langle W, \rightarrow_{1}, \ldots, \rightarrow_{n}, v\right\rangle$ if $\forall w \in W, M, w \vDash \varphi$. Then, a formula $\varphi$ is valid in DGM if $\varphi$ is true in $M$ for all $M \in \mathbf{D G M}$, and we write $\vDash_{D G M} \varphi$. A formula $\varphi$ is satisfiable in DGM if $\neg \varphi$ is not valid in DGM.

\subsubsection{Type spaces}

The formal structures mainly used in game-theoretic literature to express epistemic situations in a game are type spaces, introduced in Harsanyi (1967)-1968. The classical construction of type spaces is inductive (see Brandenburger and Dekel 1993). Each type $t_{i}$ of player $i$ is associated with an action $a_{i} \in A_{i}=X_{i}^{0}$ and represents a hierarchy of beliefs about the other players. The first level of the hierarchy is given by a probability distribution $x_{i}^{1}$ on the actions of the other players $A_{-i}=\Pi_{j \neq i} A_{j}=\Pi_{j \neq i} X_{j}^{0}=X_{-i}^{0}$, i.e., $x_{i}^{1} \in \Delta\left(X_{-i}^{0}\right)$. Call this distribution $x_{i}^{1}$ player $i$ 's 1 -order belief, and $X_{-i}^{0}$ the domain of player $i$ 's 1-order beliefs. Then, anticipating the other players' reasoning, each player $i$ will also form expectations about the other players' beliefs about the other players' action profiles. Incorporating these into the formal account results in a probability distribution $x_{i}^{2} \in \Delta\left(X_{-i}^{0} \times \Pi_{j \neq i} \Delta\left(X_{-j}^{0}\right)\right)$ over the strategy profiles and the first-order beliefs of the other players, where $X_{-i}^{0} \times \Pi_{j \neq i} \Delta\left(X_{-j}^{0}\right)=X_{-i}^{1}$ is the domain of player $i$ 's 2-order beliefs. The process goes iteratively: the domain of player $i$ 's $k+1$-order beliefs is defined as $X_{-i}^{k}=X_{-i}^{k-1} \times \Pi_{j \neq i} \Delta\left(X_{-j}^{k-1}\right)$, and a $k+1$-order belief of $i$ is a probability distribution $x_{i}^{k+1} \in \Delta\left(X_{-i}^{k}\right)$. Prima facie, if $k>h>0$ then player $i$ 's $k$-order belief is more complex than player $i$ 's $h$-order belief. It was Harsanyi's seminal contribution to see that this process catches itself at infinity, in the sense that player $i$ 's $\omega$-order belief captures player $i$ 's beliefs about the entire hierarchies of beliefs of the other players (see Brandenburger and Dekel 1993; Mertens and Zamir 1985). That is, a $\omega$-order belief of player $i$ contains information about $\omega$-order beliefs of the other players and not just about lower order beliefs of the other players. This implies that a type of player $i$ is associated with both an action of player $i$ and a probability distribution over the types of the others. Consequently, every type $t_{i} \in T_{i}$ of player $i$ can be mapped to an element of $A_{i}$ and to an element of $\Delta\left(T_{-i}\right)$, with $T_{-i}=\Pi_{j \neq i} T_{j}$. Harsanyi's characterization leads to the following simplified definition of type spaces as given by Aumann and Brandenburger (1995):

Definition 3 (Type space) A type space $\mathscr{T}$ for game $\mathbf{G}=\left\langle N,\left(A_{i}, \pi_{i}\right)_{i \in N}\right\rangle$ is a tuple $\left\langle T_{1}, \ldots, T_{n}, \beta_{1}, \ldots, \beta_{n}, \sigma_{1}, \ldots, \sigma_{n}\right\rangle$, where:

$-T_{i}$ is a countable set of types of player $i$;

$-\beta_{i}: T_{i} \longrightarrow \Delta\left(T_{-i}\right)$ is the belief function of player $i$ that associates with each type $t_{i} \in T_{i}$ a probability distribution $\mu \in \Delta\left(T_{-i}\right)$ over the types of the others $T_{-i}:=\Pi_{j \neq i} T_{j}$; 
$-\sigma_{i}: T_{i} \longrightarrow A_{i}$ is the action function of player $i$ that associates an action $a_{i} \in A_{i}$ with each type $t_{i} \in T_{i}$.

An element $t \in T$ is called state, where as usual $T:=T_{1} \times \cdots \times T_{n}$. Given a state $t$, $t_{i}$ denotes the element in $t$ corresponding to player $i$.

The truth conditions for formulas of the doxastic language $\mathscr{L}_{D G L}(\mathbf{G})$ relative to a probabilistic type space $\mathscr{T}=\left\langle T_{1}, \ldots, T_{n}, \beta_{1}, \ldots, \beta_{n}, \sigma_{1}, \ldots, \sigma_{n}\right\rangle$ are given by the following clauses. Note that formulas are evaluated at a given state $t$ of a type space $\mathscr{T}$ :

- $\mathscr{T}, t \vDash p l\left(a_{i}\right)$ iff $\sigma_{i}\left(t_{i}\right)=a_{i}$;

- $\mathscr{T}, t \vDash \neg \varphi$ iff $\mathscr{T}, t \not \models \varphi$;

- $\mathscr{T}, t \vDash \varphi \wedge \psi$ iff $\mathscr{T}, t \vDash \varphi$ and $\mathscr{T}, t \vDash \psi$;

$-\mathscr{T}, t \vDash B_{i} \varphi$ iff $\forall t^{\prime} \in T$ if $t_{i}^{\prime}=t_{i}$ and $\beta_{i}\left(t_{i}\right)\left(t_{-i}^{\prime}\right)>0$ then $\mathscr{T}, t^{\prime} \vDash \varphi$.

The truth condition of the doxastic operator $B_{i}$ is justified by the way the notion of belief is commonly defined in type spaces. A basic notion in the literature on type spaces is the event. An event is a subset $e \subseteq T$. An event for player $i$ is a subset $e_{-i} \subseteq T_{-i}$. Let $\beta_{i}\left(t_{i}\right)\left(e_{-i}\right)$ be the probability that type $t_{i}$ gives to the event $e_{-i}=\left\{t_{-i} \in T_{-i}: t_{-i} \in e_{-i}\right\}$, i.e.,

$$
\beta_{i}\left(t_{i}\right)\left(e_{-i}\right)=\sum_{t_{-i}^{\prime} \in e_{-i}} \beta_{i}\left(t_{i}\right)\left(t_{-i}^{\prime}\right) .
$$

Then, the event that player $i$ believes $e_{-i}$, denoted by $\mathscr{B}_{i}\left(e_{-i}\right)$, is defined in type spaces as follows:

$$
\mathscr{B}_{i}\left(e_{-i}\right)=\left\{t \in T: \beta_{i}\left(t_{i}\right)\left(e_{-i}\right)=1\right\} .
$$

Intuitively, $\mathscr{B}_{i}\left(e_{-i}\right)$ is the set of states at which player $i$ assigns probability 1 to the event $e_{-i}$. It is easy to check that $\mathscr{T}, t \vDash B_{i} \varphi$ if and only if $t \in \mathscr{B}_{i}\left(\left\{t_{-i}^{\prime} \in T_{-i}\right.\right.$ : $\left.\left.\mathscr{T},\left\langle t_{i}, t_{-i}^{\prime}\right\rangle \vDash \varphi\right\}\right)$. In other words, player $i$ believes $\varphi$ at state $t$ if and only if $t$ belongs to the set of states at which player $i$ assigns probability 1 to set of states at which $\varphi$ is true.

The notions of validity and satisfiability for formulas of $\mathscr{L}_{D G L}(\mathbf{G})$ relative to the class $\mathbf{T}$ of type spaces are defined in the standard way, as for doxastic game models.

\subsection{Correspondence}

In this section we are going to formally prove what we claimed in the beginning, namely that type spaces and doxastic game models are semantically equivalent with respect to the language $\mathscr{L}_{D G L}(\mathbf{G})$. The proof is developed in many steps and it will proceed as follows. Firstly, we present a way to transform type spaces into doxastic

\footnotetext{
${ }^{1}$ We are considering complete information games: the game structure and the payoff functions are common knowledge among the players. Consequently, unlike Aumann and Brandenburger (1995) there is no need to specify a payoff function for each type.
} 
models, and in particular it turns out that the resulting doxastic models are doxastic game models. Then, we show that the transformation preserves the truth of all the formulas in $\mathscr{L}_{D G L}(\mathbf{G})$. This gives us the first half of the proof, i.e., the result that if a formula of $\mathscr{L}_{D G L}(\mathbf{G})$ is satisfiable in type spaces, then it is satisfiable in doxastic game models.

The second half of the proof will proceed symmetrically. Firstly we define a way to transform doxastic game models into type spaces, then we prove that the transformation preserves the truth of all the formulas in $\mathscr{L}_{D G L}(\mathbf{G})$. Consequently, we get the second part of the result, i.e., that if a formula of $\mathscr{L}_{D G L}(\mathbf{G})$ is satisfiable in doxastic game models, then it is satisfiable in type spaces.

Starting from a given type space $\mathscr{T}=\left\langle T_{1}, \ldots, T_{n}, \beta_{1}, \ldots, \beta_{n}, \sigma_{1}, \ldots, \sigma_{n}\right\rangle$ we can transform it into a doxastic game model $M^{\mathscr{T}}=\left\langle W, \rightarrow_{1}, \ldots, \rightarrow_{n}, v\right\rangle$ where:

- $W=T$ is the set of worlds (unless differently specified, to ease the notation in what follows we simply write $w \in W$ for the world corresponding to state $t \in T$, $w^{\prime}$ for the world corresponding to state $t^{\prime}, w^{\prime \prime}$ for the world corresponding to state $t^{\prime \prime}$, and so on);

$-v: W \longrightarrow \mathscr{P}(\Phi)$ is the valuation function, defined such that $p l\left(a_{i}\right) \in v(w)$ iff $\sigma_{i}\left(t_{i}\right)=a_{i}$

$-\rightarrow_{i}$ is the belief relation of player $i$, defined as follows: $w \rightarrow_{i} w^{\prime}$ iff $\beta_{i}\left(t_{i}\right)\left(t_{-i}^{\prime}\right)>$ 0 and $t_{i}=t_{i}^{\prime}$.

We have now to prove that any model obtained via this transformation is a doxastic game model. It amounts to showing that any $M^{\mathscr{T}}$ obtained via the transformation satisfies AVC and ExIC.

Proposition $1 M^{\mathscr{T}}$ is a doxastic game model.

Proof It is straightforward to show that $M^{\mathscr{T}}$ is a doxastic model, i.e., that $M^{\mathscr{T}}$ satisfies seriality, transitivity and Euclideaness. Then we prove that $M^{\mathscr{T}}$ satisfies AVC and ExIC.

(AVC). Since $\forall i \in N, \sigma_{i}\left(t_{i}\right)=a_{i}$ for a given $a_{i} \in A_{i}$ and $\neg \exists a_{i}^{\prime} \in A_{i}$ s.t. $a_{i}^{\prime} \neq$ $a_{i}$ and $\sigma_{i}\left(t_{i}\right)=a_{i}^{\prime}$, we have that $M^{\mathscr{T}}, w \vDash p l\left(a_{i}\right)$ and $\forall a_{i}^{\prime} \in A_{i}$ s.t. $a_{i}^{\prime} \neq$ $a_{i}, M^{\mathscr{T}}, w \not \models p l\left(a_{i}^{\prime}\right)$. Then, for all $w \in W v_{i}(w)$ is a singleton and AVC is satisfied. (ExIC). In $M^{\mathscr{T}}$ we have a world $w \in W$ corresponding to each state $t \in T$. It follows by definition of $M^{\mathscr{T}}$ that if $w^{\prime} \in \rightarrow_{i}(w)$ then $t_{i}=t_{i}^{\prime}$. Furthermore, since each type $t_{i}$ is associated with a unique action $a_{i} \in A_{i}$ it follows that if $w \rightarrow_{i} w^{\prime}$ and $p l\left(a_{i}\right) \in v(w)$ then $p l\left(a_{i}\right) \in v\left(w^{\prime}\right)$. Hence ExIC is satisfied.

One might now wonder why we presented such a transformation among the many possible ones. For instance, a plausible alternative could be to transform the probability distribution into a qualitative ordering $\preceq$ in the following way: $w \preceq_{i}$ $w^{\prime}$ iff $\beta_{i}\left(t_{i}^{\prime}\right)\left(t_{-i}^{\prime}\right) \geq \beta_{i}\left(t_{i}\right)\left(t_{-i}\right)$ and $t_{i}=t_{i}^{\prime}$. The reason for our choice, as we are going to show in order to conclude the first half of the proof, is that our transformation preserves the truth of all the formulas in our language $\mathscr{L}_{D G L}(\mathbf{G})$. It means that the two structures $\mathscr{T}$ and $M^{\mathscr{T}}$ express the same epistemology with respect to the game $\mathbf{G}$ taken into account. We can now formally prove this result. 
Theorem 1 Let $t \in T$ be a state in the type space $\mathscr{T}$ and $w \in W$ the corresponding world in the doxastic game model $M^{\mathscr{T}}$ built from $\mathscr{T}$. For any $\varphi$ in $\mathscr{L}_{D G L}(\boldsymbol{G})$, if $\mathscr{T}, t \vDash \varphi$ then $M^{\mathscr{T}}, w \vDash \varphi$.

Proof By induction on the structure of $\varphi$. We prove only some cases.

Induction basis: $\left(\varphi=p l\left(a_{i}\right)\right)$. Suppose $\mathscr{T}, t \vDash p l\left(a_{i}\right)$. Then, by definition of $v$ we have that $M^{\mathscr{T}}, w \vDash p l\left(a_{i}\right)$.

Inductive steps: $\left(\varphi=B_{i} \psi\right)$. Suppose $\mathscr{T}, t \vDash B_{i} \psi$. Then, $\forall t^{\prime}$ s.t. $t_{i}^{\prime}=t_{i}$ and $\beta_{i}\left(t_{i}\right)\left(t_{-i}^{\prime}\right)$ $>0, \mathscr{T}, t^{\prime} \vDash \psi$. By inductive hypothesis, $M^{\mathscr{T}}, w^{\prime} \vDash \psi, \forall w$ such that $w \rightarrow_{i} w^{\prime}$, hence $M^{\mathscr{T}}, w \vDash B_{i} \psi$.

The second half of the proof consists in the other direction: given an arbitrary doxastic game model $M$ it is always possible to associate with it a corresponding type space $\mathscr{T}^{M}$. Furthermore, it holds that the doxastic game model $M$ and the associated type space $\mathscr{T}^{M}$ are semantically equivalent with respect to the language $\mathscr{L}_{D G L}(\mathbf{G})$.

Let us be given an arbitrary doxastic game model $M=\left\langle W, \rightarrow_{1}, \ldots, \rightarrow_{n}, v\right\rangle$. Firstly, we define the types in $\mathscr{T}^{M}$ in the following way: for all $w \in W$ we associate a type $t_{i}$ of player $i$ such that if $\rightarrow_{i}(w)=\rightarrow_{i}\left(w^{\prime}\right)$ then $\rightarrow_{i}(w)$ and $\rightarrow_{i}\left(w^{\prime}\right)$ represent the same type. Formally, $T_{i}=\left\{\rightarrow_{i}(w): w \in W\right\}$. Then for any given world $w$ in $M$ we have a state $t=\left(t_{1}, \ldots, t_{n}\right)$ in $\mathscr{T}^{M}$, defined by $\rightarrow_{1}(w), \ldots, \rightarrow_{n}(w)$. We call $t$ the state corresponding to world $w$. Secondly, we associate with each type $t_{i}$ an action $a_{i}$ specified by $v: \sigma_{i}\left(t_{i}\right)=a_{i}$ iff $p l\left(a_{i}\right) \in v(w)$. By ExIC each type will be associated with a unique action. Finally, we define the probability distribution $\beta_{i}\left(t_{i}\right)$ over $T_{-i}$ by distinguishing two cases: the case in which the support $\operatorname{supp}_{t_{i}}\left(\beta_{i}\right)=\left\{t_{-i}^{\prime} \in T_{-i}\right.$ : $\left.w \rightarrow_{i} w^{\prime}\right\}$ is finite and the case in which $\operatorname{supp}_{t_{i}}\left(\beta_{i}\right)$ is infinite. Let us define first the finite case. For each $i \in N$, for each $t_{i} \in T_{i}$ and for each $t_{-i}^{\prime} \in T_{-i}$, if $\operatorname{supp}_{t_{i}}$ is finite then:

$$
\beta_{i}\left(t_{i}\right)\left(t_{-i}^{\prime}\right)= \begin{cases}\frac{1}{\left|\operatorname{supp}_{t_{i}}\left(\beta_{i}\right)\right|} & \text { if } t_{-i}^{\prime} \in \operatorname{supp}_{t_{i}}\left(\beta_{i}\right) \\ 0 & \text { else }\end{cases}
$$

Let us now define the infinite case. For each $i \in N$, for each $t_{i} \in T_{i}$ and for each $t_{-i}^{\prime} \in T_{-i}$, if $\operatorname{supp}_{t_{i}}$ is infinite then:

$$
\beta_{i}\left(t_{i}\right)\left(t_{-i}^{\prime}\right)= \begin{cases}\frac{1}{2^{f\left(t_{-i}^{\prime}\right)}} & \text { if } t_{-i}^{\prime} \in \operatorname{supp}_{t_{i}}\left(\beta_{i}\right) \\ 0 & \text { else }\end{cases}
$$

where $f$ is a bijective function with domain $\operatorname{supp}_{t_{i}}\left(\beta_{i}\right)$ and codomain $\mathbb{N}^{+}=\{1,2, \ldots\}$. Since $\operatorname{supp}_{t_{i}}\left(\beta_{i}\right)$ is countably infinite this function is well-defined and clearly $\Sigma_{t_{-i}^{\prime} \in \operatorname{supp}_{t_{i}}\left(\beta_{i}\right)} \frac{1}{2^{f\left(t_{-i}^{\prime}\right)}}$ sums up to 1 .

To sum up, given an arbitrary doxastic game model $M=\left\langle W, \rightarrow_{1}, \ldots, \rightarrow_{n}, v\right\rangle$, we can associate with it the corresponding type space $\mathscr{T}^{M}=\left\langle T_{1}, \ldots, T_{n}, \beta_{1}, \ldots, \beta_{n}, \sigma_{1}\right.$, $\left.\ldots, \sigma_{n}\right\rangle$ defined as follows:

- a type $t_{i}$ of player $i$ for each $\rightarrow_{i}(w): T_{i}=\left\{\rightarrow_{i}(w): w \in W\right\}$; 
- an action $a_{i}$ of $t_{i}$ specified by $v: \sigma_{i}\left(t_{i}\right)=a_{i}$ iff $p l\left(a_{i}\right) \in v(w)$;

- a belief function $\beta_{i}$ defined as above.

Theorem 2 Let $w \in W$ be a world of $M$ and $t \in T$ the corresponding state in the type space $\mathscr{T}^{M}$ built from $M$. For any $\varphi$ in $\mathscr{L}_{D G L}(\boldsymbol{G})$, if $M, w \vDash \varphi$ then $\mathscr{T}^{M}, t \vDash \varphi$.

Proof By induction on the structure of $\varphi$. We prove only some cases.

Induction basis: $\left(\varphi=p l\left(a_{i}\right)\right)$. Suppose $M, w \vDash p l\left(a_{i}\right)$. Then, by definition of $v$ we have that $\mathscr{T}^{M}, t \vDash p l\left(a_{i}\right)$.

Inductive steps: $\left(\varphi=B_{i} \psi\right)$. Suppose $M, w \vDash B_{i} \psi$. Consequently, $M, w^{\prime} \vDash$ $\psi, \forall w^{\prime} \in \rightarrow_{i}(w)$. By inductive hypothesis, $\forall t^{\prime}$ s.t. $t_{i}^{\prime}=t_{i}$ and $\beta_{i}\left(t_{i}\right)\left(t_{-i}^{\prime}\right)>$ $0, \mathscr{T}^{M}, t^{\prime} \vDash \psi$, hence $\mathscr{T}^{M}, t \vDash B_{i} \psi$.

The following corollary finally states that the class of type spaces $\mathbf{T}$ and the class of doxastic game models DGM provide equivalent semantics with respect to the language $\mathscr{L}_{D G L}(\mathbf{G})$.

Corollary 1 A formula $\varphi$ of $\mathscr{L}_{D G L}(\boldsymbol{G})$ is satisfiable in $\boldsymbol{T}$ iff it is satisfiable in $\mathbf{D G M} .^{2}$

\section{Models with belief and knowledge}

In this section we want to extend our language to deal with more than one epistemic attitude. A straightforward extension is to introduce another operator to represent "knowledge" or "absolute certainty with no possibility at all for error". We want to show if and how we can interpret it on our structures, proving the same equivalence result between type spaces and Kripke models with respect to the enriched language.

\subsection{Language}

Given a finite simultaneous-move game $\mathbf{G}=\left\langle N,\left(A_{i}, \pi_{i}\right)_{i \in N}\right\rangle$, we define an extension of our logic DGL, and we call it epistemic-doxastic game logic EDGL. The language $\mathscr{L}_{E D G L}(\mathbf{G})$ of EDGL is defined by the following grammar:

$$
\varphi::=p l\left(a_{i}\right)|\neg \varphi| \varphi \wedge \varphi\left|K_{i} \varphi\right| B_{i} \varphi \mid \square \varphi
$$

where $a_{i} \in A_{i}$ and $i \in N$.

The language $\mathscr{L}_{E D G L}(\mathbf{G})$ is the doxastic game language $\mathscr{L}_{D G L}(\mathbf{G})$ of DGL extended by the knowledge operator $K_{i}$, and the universal operator $\square$, which turns out to be useful for obtaining the axiomatization, as we will see in Sect. $4 . K_{i} \varphi$ is read as "player $i$ knows that $\varphi$ is true", while $\square \varphi$ is read as " $\varphi$ is universally true". As before, let us abbreviate the dual of $K_{i}$ as $\widehat{K}_{i} \varphi:=\neg K_{i} \neg \varphi$ and the dual of $\square$ as $\diamond \varphi:=\neg \square \neg \varphi$.

\footnotetext{
2 In order to avoid further complications, here we limit ourselves to the countable case: countable type sets and countable possible worlds set. However, our results generalize to the uncountable case by appropriately endowing type sets $T_{i}$ and $T_{-i}$ with $\sigma$-algebras, and by ensuring that every $\beta_{i}\left(t_{i}\right)$ is a measurable function, as usual in type spaces literature (see Aumann and Brandenburger 1995, Sect. 6).
} 


\subsection{Semantics}

\subsubsection{Type spaces}

In a similar way to what we did above we want to interpret our language $\mathscr{L}_{E D G L}(\mathbf{G})$ over a type space $\mathscr{T}$ as semantics. In order to do that, we simply need to add to the previous list of clauses of Sect. 2.2.2 the following clauses for $K_{i}$-formulas and $\square$-formulas:

- $\mathscr{T}, t \vDash K_{i} \varphi$ iff $\forall t^{\prime} \in T$ if $t_{i}^{\prime}=t_{i}$ then $\mathscr{T}, t^{\prime} \vDash \varphi$;

- $\mathscr{T}, t \vDash \square \varphi$ iff $\forall t^{\prime} \in T, \mathscr{T}, t^{\prime} \vDash \varphi$.

Looking at these clauses, we can observe that the $K_{i}$-operator ranges over all the states with the same type for player $i$, whereas the $\square$-operator ranges over all the states in $\mathscr{T}$. We will spend more words in Sect. 3.5 on the interpretation. In particular, although the clause for $K_{i}$-formulas seems the obvious one for type spaces, we will see that it is far from being uncontroversial and it will have important consequences on Kripke models.

\subsubsection{Epistemic-doxastic models}

In the literature about Kripke models the semantics usually associated with epistemicdoxastic languages with a $B_{i}$-operator and a $K_{i}$-operator are epistemic-doxastic models, or just epistemic models for brevity (see Kraus and Lehmann 1988). Epistemic models are nothing but multi-relational Kripke models commonly used in modal logic (Blackburn et al. 2001).

Definition 4 (Epistemic-doxastic model) An epistemic-doxastic model is a tuple $M=\left\langle W, \sim_{1}, \ldots, \sim_{n}, \rightarrow_{1}, \ldots, \rightarrow_{n}, v\right\rangle$ where:

- $W$ is a countable set of possible worlds;

$-v: W \longrightarrow \mathscr{P}(\Phi)$ is the valuation function for the set $\Phi$ of atomic facts defined in Sect. 2.1;

$-\sim_{i}$ is the epistemic accessibility relation of player $i$, that is an equivalence relation over $W$;

- $\rightarrow{ }_{i} \subseteq W \times W$ is the belief relation of player $i$ which satisfies the following conditions:

- seriality: $\forall w \exists w^{\prime}$ s.t. $w \rightarrow_{i} w^{\prime}$;

$-\rightarrow{ }_{i} \subseteq \sim_{i}$

- $\forall w, w^{\prime} \in W$, if $w \sim_{i} w^{\prime}$ then $\rightarrow_{i}(w) \subseteq \rightarrow_{i}\left(w^{\prime}\right)$.

Moreover, let us write $\sim_{i}(w)$ for the partition cell (also called "information set") of player $i$ containing world $w: \sim_{i}(w):=\left\{w^{\prime} \in W: w \sim_{i} w^{\prime}\right\}$.

In order to interpret $\mathscr{L}_{E D G L}(\mathbf{G})$ over epistemic models and to state a result of semantic equivalence with respect to type spaces, we are going to identify a subclass of epistemic models, that we call epistemic-doxastic game models, or just epistemic game models EGM. 
Definition 5 (Epistemic game model) Epistemic game models are epistemic-doxastic models satisfying the conditions AVC and ExIC given in Definition 2, and the following condition:

- Epistemic independence condition (EIC):

$$
\sim_{1}\left(w_{1}\right) \cap \ldots \cap \sim_{n}\left(w_{n}\right) \neq \emptyset \text { for every }\left(w_{1}, \ldots, w_{n}\right) \in W^{n} .
$$

Roughly speaking, EIC says that each player has no reason to rule out any possible information set of the others specified in the model: if an information set of $i$ is present in the model, then the other players should not consider it impossible at any world.

Epistemic game models can be used to represent a semantics for the language $\mathscr{L}_{E D G L}(\mathbf{G})$. The semantic clauses are the same as for doxastic game models, plus the following clauses for $K_{i}$-formulas and $\square$-formulas:

- $M, w \vDash K_{i} \varphi$ iff $\forall w^{\prime} \in W$ if $w \sim_{i} w^{\prime}$ then $M, w^{\prime} \vDash \varphi$;

- $M, w \vDash \square \varphi$ iff $\forall w^{\prime} \in W, M, w^{\prime} \vDash \varphi$.

As for DGM, we say that a formula $\varphi$ is true in a model $M$ if $\forall w \in W, M, w \vDash \varphi$. Then, a formula $\varphi$ is valid in EGM (and we write $\vDash_{E G M} \varphi$ ) if $\varphi$ is true in $M$ for all $M \in \mathbf{E G M}$, and a formula $\varphi$ is satisfiable in EGM if $\neg \varphi$ is not valid in EGM.

We want to point out also that the condition ExIC in epistemic game models implies that each player knows her own action. This makes sense, since we are describing an ex interim stage of the game, where each player is already certain about her own choice. This is expressed by the following validity:

Lemma $1 \forall i \in N, \forall a_{i} \in A_{i}, \vDash_{E G M} \operatorname{pl}\left(a_{i}\right) \rightarrow K_{i} \operatorname{pl}\left(a_{i}\right)$.

Proof By contradiction, suppose that $M, w \vDash p l\left(a_{i}\right)$ and that $M, w \not \models K_{i} p l\left(a_{i}\right)$. Consequently, $\exists u \in \sim_{i}(w)$ s.t. $M, u \not \models p l\left(a_{i}\right)$. Hence, by AVC $\exists a_{i}^{\prime} \in A_{i}$ s.t. $a_{i}^{\prime} \neq$ $a_{i}$ and $M, u \vDash p l\left(a_{i}^{\prime}\right)$. By definition of $\rightarrow_{i}$ we have that if $w \sim_{i} u$ then $\rightarrow_{i}(w)=\rightarrow_{i}$ (u). It follows that $\forall w^{\prime} \in W$, if $w^{\prime} \in \rightarrow_{i}(w)$ then $w^{\prime} \in \rightarrow_{i}(u)$ too. Then, by ExIC $M, w^{\prime} \vDash p l\left(a_{i}\right)$ and $M, w^{\prime} \vDash p l\left(a_{i}^{\prime}\right)$. Contradiction with AVC.

\subsection{Correspondence}

In this section we prove the semantic equivalence between type spaces and epistemic game models with respect to the language $\mathscr{L}_{E D G L}(\mathbf{G})$. The proof will proceed in the same way as before, i.e., it will be divided into two parts and we will make use of a transformation of one structure into the other in order to show the equivalence.

Given an arbitrary type space $\mathscr{T}$, the corresponding epistemic-doxastic model $M^{\mathscr{T}}$ is defined as the tuple $M^{\mathscr{T}}=\left\langle W, \sim_{1}, \ldots, \sim_{n}, \rightarrow_{1}, \ldots, \rightarrow_{n}, v\right\rangle$ where:

$-W=T$ is the set of worlds;

$-v: W \longrightarrow \mathscr{P}(\Phi)$ is the valuation function such that $p l\left(a_{i}\right) \in v(w)$ iff $\sigma_{i}\left(t_{i}\right)=a_{i}$;

$-\sim_{i}$ is the accessibility relation of player $i$, given by: $\forall w, w^{\prime} \in W, w \sim_{i} w^{\prime}$ iff $t_{i}=$ $t_{i}^{\prime}$. Then $\sim_{i}$ determines a partition over $W$;

$-\rightarrow_{i}$ is the belief relation of player $i$, defined as follows: $w \rightarrow_{i} w^{\prime}$ iff $\beta_{i}\left(t_{i}\right)\left(t_{-i}^{\prime}\right)>$ 0 and $t_{i}=t_{i}^{\prime}$. 
We have now to show that the epistemic-doxastic model we obtain via the transformation is an epistemic game model, namely we have to show that it satisfies AVC, ExIC and EIC.

Proposition $2 M^{\mathscr{T}}$ is an epistemic game model.

Proof The proof for AVC and ExIC is similar to Proposition 1. Here we only prove that EIC holds too.

(EIC). Since $T=T_{1} \times \cdots \times T_{n}$, each state $t \in T$ has the form $\left(t_{1}, \ldots, t_{n}\right)$, and $\forall t_{i} \forall t_{-i} \exists t$ s.t. $t=\left(t_{i}, t_{-i}\right)$. Denoting by $\sim_{i}\left(t_{i}\right)$ the partition cell corresponding to type $t_{i}$, it follows that $\forall\left(t_{1}, \ldots, t_{n}\right) \in T \exists w \in W$ s.t. $\sim_{1}\left(t_{1}\right) \cap \cdots \cap \sim_{n}\left(t_{n}\right)=w$. Consequently, $\sim_{1}\left(w_{1}\right) \cap \cdots \cap \sim_{n}\left(w_{n}\right) \neq \emptyset$ for every $\left(w_{1}, \ldots, w_{n}\right) \in W^{n}$ and EIC is satisfied.

To conclude the first half of the proof we show that this transformation into epistemic game models preserves the truth of all the formulas of $\mathscr{L}_{E D G L}(\mathbf{G})$. The proof is similar to the previous one, so in this section we can just focus on the part for $K_{i}$-formulas and $\square$-formulas.

Theorem 3 Let $t \in T$ be a state in $\mathscr{T}$ and let $w \in W$ be the corresponding world in $M^{\mathscr{T}}$. For any $\varphi$ in $\mathscr{L}_{E D G L}(\boldsymbol{G})$, if $\mathscr{T}, t \vDash \varphi$ then $M^{\mathscr{T}}, w \vDash \varphi$.

Proof By induction on the structure of $\varphi$.

$\left(\varphi=K_{i} \psi\right)$. Suppose $\mathscr{T}, t \vDash K_{i} \psi$. Then, $\forall t^{\prime}$ s.t. $t_{i}^{\prime}=t_{i} \mathscr{T}, t^{\prime} \vDash \psi$. By inductive hypothesis, $M^{\mathscr{T}}, w^{\prime} \vDash \psi, \forall w^{\prime} \in \sim_{i}(w)$, hence $M^{\mathscr{T}}, w \vDash K_{i} \psi$.

$(\varphi=\square \psi)$. Suppose $\mathscr{T}, t \vDash \square \psi$. Then, $\forall t^{\prime} \in T, \mathscr{T}, t^{\prime} \vDash \psi$. By inductive hypothesis, $M^{\mathscr{T}}, w^{\prime} \vDash \psi, \forall w^{\prime} \in W$, hence $M^{\mathscr{T}}, w \vDash \square \psi$.

In the second part of the proof we are going to show the other direction: given an arbitrary epistemic game model $M$ it is always possible to associate with it a corresponding type space $\mathscr{T}^{M}$. Moreover, it holds again that the epistemic game model $M$ and the associated type space $\mathscr{T}^{M}$ are semantically equivalent with respect to the language $\mathscr{L}_{E D G L}(\mathbf{G})$.

Let us be given an arbitrary epistemic game model $M$. Firstly, we define a type $t_{i} \in T_{i}$ for each $i$ 's partition cell $\sim_{i}(w)$ in $M: T_{i}=\left\{\sim_{i}(w): w \in W\right\}$. Notice that when we have $\sim_{i}$ relations in the model this is equivalent to the definition of types given for Theorem 2. Then by EIC, given $n$ arbitrary partition cells, one for each player, the intersection will always be non-empty. The worlds $w$ in the intersection are associated with the state $t=\left(t_{1}, \ldots, t_{n}\right)$, where types $t_{1}, \ldots, t_{n}$ are determined by the partition cells $\sim_{i}(w)$ for all $i$ : let us call $t$ the state corresponding to those worlds $w$ in the intersection. Moreover, EIC guarantees that in $\mathscr{T}^{M}$ the states $T$ correspond to the Cartesian product of type sets $T_{i}$, i.e. $T=T_{1} \times \cdots \times T_{n}$. Secondly, we associate with each type $t_{i}$ an action $a_{i}$ specified by $v: \sigma_{i}\left(t_{i}\right)=a_{i}$ iff $p l\left(a_{i}\right) \in v(w)$. By ExIC each type will be associated with a unique action. Finally, for each type $t_{i}$ we define the probability distribution $\beta_{i}\left(t_{i}\right)$ over $T_{-i}$ in the same way as we did in the proof of Theorem 2 by distinguishing two cases, the case in which the support $\operatorname{supp}_{t_{i}}\left(\beta_{i}\right)=\left\{t_{-i}^{\prime} \in T_{-i}: w \rightarrow_{i} w^{\prime}\right\}$ is finite and the case in which it is infinite, with $w$ being the world in $W$ associated with the type $t_{i}$. 
To sum up, taken an arbitrary $M=\left\langle W, \sim_{1}, \ldots, \sim_{n}, \rightarrow_{1}, \ldots, \rightarrow_{n}, v\right\rangle$, we can associate with it the corresponding type space $\mathscr{T}^{M}=\left\langle T_{1}, \ldots, T_{n}, \beta_{1}, \ldots, \beta_{n}, \sigma_{1}\right.$, $\left.\ldots, \sigma_{n}\right\rangle$ defined as follows:

- a type $t_{i}$ of player $i$ for each $i$ 's partition cell $\sim_{i}(w)$ in $M: T_{i}=\left\{\sim_{i}(w): w \in W\right\}$;

- an action $a_{i}$ of $t_{i}$ specified by $v: \sigma_{i}\left(t_{i}\right)=a_{i}$ iff $p l\left(a_{i}\right) \in v(w)$;

- a belief function $\beta_{i}$ defined differently for the finite case and for the infinite case, as before.

Theorem 4 Let $w \in W$ be a world of $M$ and $t \in T$ the corresponding state in $\mathscr{T}^{M}$. For any $\varphi$ in $\mathscr{L}_{E D G L}(\boldsymbol{G})$, if $M, w \vDash \varphi$ then $\mathscr{T}^{M}, t \vDash \varphi$.

Proof By induction on the structure of $\varphi$.

$\left(\varphi=K_{i} \psi\right)$. Suppose $M, w \vDash K_{i} \psi$. Then, by definition $M, w^{\prime} \vDash \psi, \forall w^{\prime} \in \sim_{i}(w)$. By inductive hypothesis, $\forall t^{\prime}$ s.t. $t_{i}^{\prime}=t_{i}, \mathscr{T}^{M}, t^{\prime} \vDash \psi$, hence $\mathscr{T}, t \vDash K_{i} \psi$.

$(\varphi=\square \psi)$. Suppose $M, w \vDash \square \psi$. Then, by definition $M, w^{\prime} \vDash \psi, \forall w^{\prime} \in W$. By inductive hypothesis, $\forall t^{\prime} \in T, \mathscr{T}^{M}, t^{\prime} \vDash \psi$, hence $\mathscr{T}^{M}, t \vDash \square \psi$.

The following corollary then states that the class of type spaces $\mathbf{T}$ and the class of epistemic game models EGM provide equivalent semantics with respect to the language $\mathscr{L}_{E D G L}(\mathbf{G})$.

Corollary 2 A formula $\varphi$ of $\mathscr{L}_{E D G L}(\boldsymbol{G})$ is satisfiable in $\boldsymbol{T}$ iff it is satisfiable in $\mathbf{E G M}$.

\subsection{Examples}

We consider a two player game $\mathbf{G}=\left\langle A n n, B o b ; A_{A}=\{U, D\}, \pi_{A} ; A_{B}=\right.$ $\left.\{L, R\}, \pi_{B}\right\rangle$. Then, an example of type space for $\mathbf{G}$ is pictured in Fig. 1 .

Figures 2 and 3 depict the transformation into epistemic game models as defined above. Arrows represent the belief relation: an arrow going from $w$ to $w^{\prime}$ means $w \rightarrow_{i} w^{\prime}$. Coloured squares that partition the set of all possible worlds represent the accessibility relation and each square corresponds to a type.

Notice that if we drop the ex interim condition we can represent a situation in which players have not decided yet their actions and hence they do not know their future action (Fig. 4). Indeed, Fig. 4 pictures an information set of Ann that contains

Fig. 1 A possible type space

\begin{tabular}{|c|c|c|c|c|}
\hline & & & & \\
\hline & $1 L$ & $2 L$ & $1 R$ & $2 R$ \\
\hline $1 U$ & $\frac{1}{2}, \frac{1}{3}$ & $\frac{1}{2}, 1$ & $0, \frac{1}{3}$ & $0, \frac{1}{4}$ \\
\hline $2 U$ & $\frac{1}{3}, 0$ & $\frac{2}{3}, 0$ & 0,0 & 0,0 \\
\hline $1 D$ & $\frac{1}{2}, 0$ & $\frac{1}{2}, 0$ & 0,0 & $0, \frac{3}{4}$ \\
\hline $2 D$ & $\frac{1}{2}, \frac{2}{3}$ & 0,0 & $\frac{1}{2}, \frac{2}{3}$ & 0,0 \\
\hline
\end{tabular}


Fig. 2 Transformation: Ann

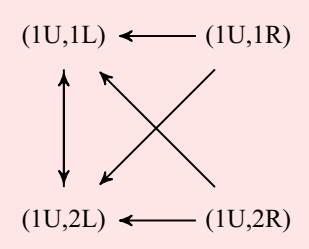

(2U,1L) (2U,1R)

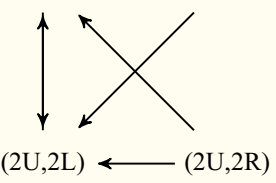

Fig. 3 Transformation: Bob

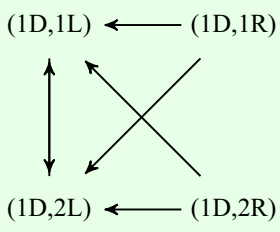

$(2 \mathrm{D}, 1 \mathrm{~L}) \longleftrightarrow(2 \mathrm{D}, 1 \mathrm{R})$

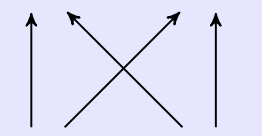

(2D,2L)

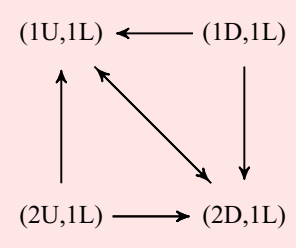

$(1 \mathrm{U}, 1 \mathrm{R}) \longleftarrow(1 \mathrm{D}, 1 \mathrm{R})$

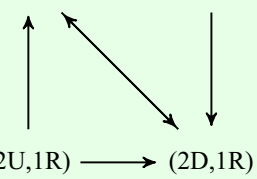

(1U,2L)

(1D,2L)

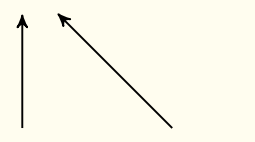

(2U,2L)

(2D,2L)

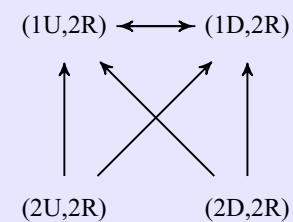

both worlds where her action is $U$ and worlds where her action is $D$ : this means that Ann is still uncertain about her choice of action.

\subsection{Discussion}

After having presented the equivalence results between the Kripkean semantics and type space semantics for the qualitative epistemic languages of belief and knowledge, we want to talk about two conceptual issues that are relevant here. First of all, we spend some more words on the concept of knowledge introduced in Sect. 3. Secondly, we briefly discuss the distinction between probabilistic (quantitative) type spaces and qualitative type spaces.

\subsubsection{On the knowledge operator}

One might wonder why we introduced the operator $K_{i}$. In fact, only one basic epistemic operator is normally introduced and used in type spaces: the probability 1-operator, 
Fig. 4 Transformation without ExIC: Ann type 1

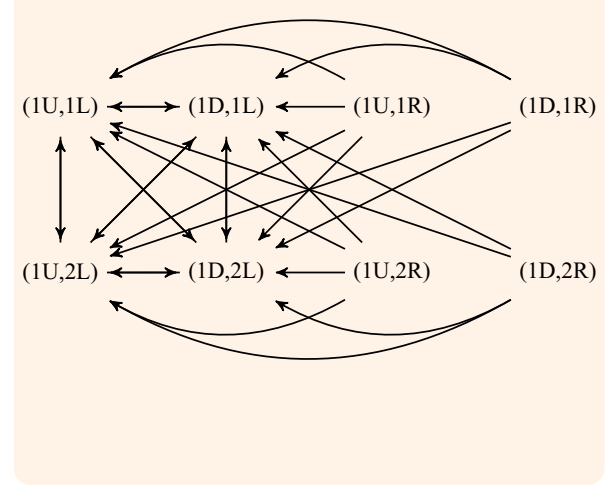

that corresponds to the operator $B_{i}$. In economic literature this probability 1-operator is sometimes called knowledge and sometimes belief (see Aumann and Brandenburger 1995; Brandenburger 2008). Indeed, Aumann and Brandenburger (1995) write:

In this paper, "know" means "ascribe probability 1 to". This is sometimes called "believe", while "know" is reserved for absolute certainty with no possibility at all for error.

The reason why we introduced two different epistemic operators is related to that observation. As mentioned in the previous quote, in (modal) epistemic literature there are two different basic notions, knowledge and belief, that normally correspond to a S5-operator and a KD45-operator. Then, it makes sense to try to express these two different epistemic attitudes in both the structures we are dealing with, and to compare their interpretations in parallel.

The most obvious thing to do if we want to represent in type spaces both epistemic attitudes, absolute certainty with no possibility for error and probability 1 (or, knowledge and belief), is to interpret the knowledge operator as we showed in Sect. 3.2.1. Far from being uncontroversial, this definition of a knowledge operator on type spaces gives rise to interesting properties that make the $K_{i}$-operator stronger than the classical S5-knowledge operator used in computer science and distributed artificial intelligence (Fagin et al. 1995). This means that all S5-axioms still hold for the $K_{i}$-operator, but $K_{i}$ also satisfies some additional properties. Although in the single agent case the S5-knowledge operator and the $K_{i}$-operator coincide, the two clearly diverge in the multi-agent case. As we will immediately see, the main difference from the standard knowledge operator is made by condition EIC. The following propositions show two properties satisfied by the $K_{i}$-operator but not by the classical S5-knowledge operator.

Proposition 3 For all $i, j \in N$ such that $i \neq j$, we have $\vDash_{E G M} K_{i} K_{j} \varphi \leftrightarrow \square \varphi$.

Proof (right-to-left). Let $i \neq j$. Suppose that $M, w \vDash K_{i} K_{j} \varphi$. Then, $\forall w^{\prime} \in \sim_{i}(w)$, $M, w^{\prime} \vDash K_{j} \varphi$. However, every world $u \in W$ belongs to a partition cell of player 
$j$, i.e., $\sim_{j}(u)$, and, by EIC, for every partition cell $\sim_{j}(u)$ of player $j$ we have that $\sim_{j}(u) \cap \sim_{i}(w) \neq \emptyset$. Then, in every partition cell $\sim_{j}(u)$ of player $j$ there is a world $w^{\prime}$ s.t. $M, w^{\prime} \vDash K_{j} \varphi$. It means that $\forall u \in W, M, u \vDash \varphi$. Hence, $M, w \vDash \square \varphi$. (left-to-right). Trivial.

Proposition 4 Let $p l\left(a_{-i}\right)$ be defined as $p l\left(a_{-i}\right):=\bigwedge_{j \neq i} p l\left(a_{j}\right)$. Then, for all $i \in N$, we have $\vDash_{E G M} \diamond p l\left(a_{-i}\right) \leftrightarrow \widehat{K}_{i} p l\left(a_{-i}\right)$.

Proof (left-to-right). Suppose that $M, w \vDash \diamond p l\left(a_{-i}\right)$. Then, $\exists w^{\prime} \in W$ s.t. $M, w^{\prime} \vDash$ $p l\left(a_{-i}\right)$. For each $j \in N$ s.t. $j \neq i$ and for each $p l\left(a_{j}\right) \in p l\left(a_{-i}\right)$, by ExIC there must be a partition cell $\sim_{j}\left(w^{\prime}\right)$ where $\forall u \in \sim_{j}\left(w^{\prime}\right), M, u \vDash p l\left(a_{j}\right)$. By EIC, we have that: $\forall u^{\prime} \in W, \sim_{i}\left(u^{\prime}\right) \cap \bigcap_{j \neq i} \sim_{j}\left(w^{\prime}\right) \neq \emptyset$. Hence, $M, w \vDash \widehat{K}_{i} p l\left(a_{-i}\right)$.

(right-to-left). Trivial.

Proposition 4 says that if a particular action profile $p l\left(a_{-i}\right)$ is possible, i.e., if $p l\left(a_{-i}\right)$ holds at some world in the model, then player $i$ knows for sure that it is possible. Proposition 3 on the other hand is a more general and stronger property of the model: it states that if $i$ and $j$ are different players, then player $i$ knows that player $j$ knows that $\varphi$ if and only if $\varphi$ is necessary.

Let us spend some words on the meaning of Proposition 3. As it is clear from the definition of type space given in Sect. 2.2.2, a type for player $i$ provides information about the 'psychological situation' of player $i$, namely: (i) her actual choice, and (ii) her subjective probability distribution over possible states. As emphasized above, the operator $K_{i}$ should be interpreted as an operator of absolute unrevisable certainty, in the sense that $K_{i} \varphi$ is true if and only if $\varphi$ is true in all states that player $i$ envisages (or imagines) as possible. The truth condition of this operator in the type space semantics given in Sect. 3.2.1 presupposes that for every possible psychological situation of the other players, player $i$ envisages a state in which this psychological situation occurs. In other words, player $i$ only excludes from her information set those states in which her psychological situation is different from her actual psychological situation. Under this assumption, Proposition 3 makes perfect sense. Indeed, let $i$ and $j$ be different players. The previous assumption implies that, for every possible state $t^{\prime \prime} \in T$ that is not in the information set of player $i$ at the actual state $t$, there exists a state $t^{\prime} \in T$ in player $i$ 's information set at the actual state $t$ such that $t^{\prime \prime}$ is included in player $j$ 's information set at state $t^{\prime}$. Therefore, clearly, if $K_{i} K_{j} \varphi$ is true at state $t$ then $\square \varphi$ is true at $t$ too. The other direction of the equivalence (i.e., $\square \varphi$ implies $K_{i} K_{j} \varphi$ ) holds for obvious reasons.

\subsubsection{Qualitative vs. quantitative type spaces}

In Sect. 2.2.2 we have introduced probabilistic type spaces as defined by Aumann and Brandenburger (1995) and justified this definition on the basis of Harsanyi's characterization. It is worth noting that Harsanyi's characterization only holds because of the properties of probabilities. In particular, in Harsanyi's type spaces probabilities are $\sigma$-additive, and therefore continuous on increasing and decreasing sequences of events. As shown by Fagin et al. (1999) (see also Fagin 1994; Fagin et al. 1991; Heifetz and Samet 1998), there is an analogous inductive construction of qualitative type spaces that does not satisfy the property that all information about other players' 
beliefs is captured at level $\omega$, as the construction might need to carry out transfinitely long. Type spaces studied by Fagin et al. are qualitative: given player $i$ 's basic domain of uncertainty $W_{i}^{0}=A_{-i}, f_{0}$ denotes a member of $W_{i}^{0}$. Each assignment $\left(f_{0}\right)$ represents a "possible 1-world", and the domain of $i$ 's 1-order beliefs is the set of all possible 1-worlds $W_{i}^{1}$. Then, $i$ 's 1 -order belief is defined as a set $f_{1}(i) \subseteq W_{i}^{1}$. Inductively, player $i$ 's $k$-level belief is defined as a set $f_{k}(i) \subseteq W_{i}^{k}$ of possible k-worlds, i.e., k-tuples of functions $\left(f_{0}, \ldots, f_{k-1}\right)$. Equivalently, a 1-order belief of $i$ can be expressed as a function $f_{1}(i): W_{i}^{1} \rightarrow\{0,1\}$, and $i$ 's k-order belief as a function $f_{k}(i): W_{i}^{1} \times \cdots \times W_{i}^{k} \rightarrow\{0,1\}$. Specifically, it is shown by Fagin et al. that for qualitative type spaces it is not necessarily the case that a $\omega$-order belief $f_{\omega}(i)$ of $i$ contains information about $\omega$-order beliefs of the other players. In this sense, there is no simplified definition of qualitative type spaces which is analogous to Definition 3 in Sect. 2.2.2 for quantitative type spaces and which is justified in the light of an argument à la Harsanyi. However, since we do not use such an inductive construction of qualitative hierarchies of beliefs and we deal with finitary modal logics, the results by Fagin et al. are not problematic from our standpoint.

The reason why we have introduced probabilistic (quantitative) type spaces instead of qualitative type spaces à la Fagin et al. is that in Sect. 6 we will move from a qualitative representation of epistemic attitudes to a quantitative representation which requires a probabilistic interpretation in terms of probabilistic type spaces. Thus, we preferred to state semantic equivalence with respect to a unique type space representation that applies both to qualitative and quantitative languages. However, it is worth noting that, as for the interpretation of the qualitative epistemic language with belief operators given in Sect. 2 and of its extension by knowledge operators given in Sect. 3, we could have completely omitted the probabilistic aspect of type spaces as defined in Definition 3 and given an analogous qualitative definition in which functions $\beta_{i}$ are replaced by functions $\beta_{i}^{\prime}: T_{i} \longrightarrow 2^{T_{-i}}$, where $t_{-i}^{\prime} \in \beta_{i}^{\prime}\left(t_{i}\right)$ means that player $i$ 's type $t_{i}$ considers type $t_{-i}^{\prime}$ possible and, viceversa, $t_{-i}^{\prime} \notin \beta_{i}^{\prime}\left(t_{i}\right)$ means that player $i$ 's type $t_{i}$ considers type $t_{-i}^{\prime}$ impossible. The two semantics, the one with functions $\beta_{i}$ of Definition 3 and the one in which functions $\beta_{i}$ are replaced by functions $\beta_{i}^{\prime}$, have clearly the same sets of validities for both qualitative epistemic languages introduced here. We conjecture that the sets of validities for the two qualitative epistemic languages do not change if we adopt the qualitative type space semantics à la Fagin et al. in which type spaces are defined in an inductive way. We postpone the proof of this conjecture to future work.

\section{Axiomatization}

In this section we provide sound and complete axiomatizations for the logics EDGL and DGL relative to the class of epistemic game models and doxastic game models, respectively.

Given the equivalences between epistemic game models and type spaces with respect to EDGL (Corollary 3) and between doxastic game models and type spaces with respect to DGL (Corollary 1), these axiomatizations will also turn out to be sound and complete relative to type spaces. 
- Axioms for EDGL:

(1) All tautologies of classical propositional logic

(2) Axioms K, T, 4 and B for the universal modality $\square$

(a) $\square(\varphi \rightarrow \psi) \rightarrow(\square \varphi \rightarrow \square \psi)$

(b) $\square \varphi \rightarrow \varphi$

(c) $\square \varphi \rightarrow \square \square \varphi$

(d) $\varphi \rightarrow \square \diamond \varphi$

(3) Axioms K, T, 4 and B for the knowledge modality $K_{i}$

(a) $K_{i}(\varphi \rightarrow \psi) \rightarrow\left(K_{i} \varphi \rightarrow K_{i} \psi\right)$

(b) $K_{i} \varphi \rightarrow \varphi$

(c) $K_{i} \varphi \rightarrow K_{i} K_{i} \varphi$

(d) $\varphi \rightarrow K_{i} \widehat{K}_{i} \varphi$

(4) Axioms $\mathrm{K}$ and $\mathrm{D}$ for the belief modality $B_{i}$

(a) $B_{i}(\varphi \rightarrow \psi) \rightarrow\left(B_{i} \varphi \rightarrow B_{i} \psi\right)$

(b) $\neg\left(B_{i} \varphi \wedge B_{i} \neg \varphi\right)$

(5) Interaction axioms between universal modality, knowledge modality and belief modality

(a) $\square \varphi \rightarrow K_{i} \varphi$

(b) $K_{i} \varphi \rightarrow B_{i} \varphi$

(c) $B_{i} \varphi \rightarrow K_{i} B_{i} \varphi$

(d) $\left(\diamond K_{1} \varphi_{1} \wedge \ldots \wedge \diamond K_{n} \varphi_{n}\right) \rightarrow \diamond\left(K_{1} \varphi_{1} \wedge \ldots \wedge K_{n} \varphi_{n}\right)$

(6) Axioms for the atomic formulas $p l\left(a_{i}\right)$

(a) $\bigvee_{a_{i} \in A_{i}} \operatorname{pl}\left(a_{i}\right)$

(b) $p l\left(a_{i}\right) \rightarrow \neg p l\left(a_{i}^{\prime}\right)$ if $a_{i} \neq a_{i}^{\prime}$

(c) $p l\left(a_{i}\right) \rightarrow B_{i} p l\left(a_{i}\right)$

- Rules of inference for EDGL:

(7) From $\varphi$ and $\varphi \rightarrow \psi$ infer $\psi$

(8) From $\varphi$ infer $\square \varphi$

Fig. 5 Axiomatization of EDGL

Theorem 5 The set of validities of the logic EDGL relative to the class of epistemic game models (EGM) is completely axiomatized by the principles given in Fig. 5.

Proof Proving that the axioms given in Fig. 5 are sound with respect to the class EGM and that the inference rules preserve validity is just a routine task and we do not give it here.

As to completeness, let us define the class of weak epistemic game models (WEGM) as the class of epistemic models that satisfy the epistemic independence condition (EIC) but do not necessarily satisfy the adequate valuation condition (AVC) and the ex interim condition (ExIC). In other words, epistemic game models are a subclass of weak epistemic game models that satisfy both the adequate valuation condition (AVC) and the ex interim condition (ExIC).

We write $\vDash_{W E G M} \varphi$ to mean that the EDGL-formula $\varphi$ is valid relative to the class WEGM.

Moreover, for any finite set $\Delta$ of EDGL-formulas, we write $\Delta \vDash_{W E G M} \varphi$ to mean that $\varphi$ is a logical consequence of the set of formulas $\Delta$ relative to the class WEGM. That is, $\Delta \vDash_{W E G M} \varphi$ iff, for every weak epistemic game model $M=\left\langle W, \sim_{1}, \ldots, \sim_{n}\right.$ $\left., \rightarrow_{1}, \ldots, \rightarrow_{n}, v\right\rangle$, if $M, w \vDash \bigwedge_{\psi \in \Delta} \psi$ for all $w \in W$, then $M, w \vDash \varphi$ for all $w \in W$.

The following Proposition 5 highlights that the validity problem relative to the class EGM is reducible to the logical consequence problem relative to the class WEGM. 


\section{Proposition 5 Let}

$$
\begin{aligned}
& \Delta_{0}=\left\{\bigvee_{a_{i} \in A_{i}} p l\left(a_{i}\right): i \in N\right\} \cup \\
&\left\{p l\left(a_{i}\right) \rightarrow \neg p l\left(a_{i}^{\prime}\right): i \in N \text { and } a_{i}, a_{i}^{\prime} \in A_{i} \text { with } a_{i} \neq a_{i}^{\prime}\right\} \cup \\
&\left\{p l\left(a_{i}\right) \rightarrow B_{i} p l\left(a_{i}\right): i \in N \text { and } a_{i} \in A_{i}\right\}
\end{aligned}
$$

Then, for every EDGL-formula $\varphi, \vDash_{E G M} \varphi$ iff $\Delta_{0} \vDash_{W E G M} \varphi$.

Proof We just need to observe that the (global) axioms in $\Delta_{0}$ force a weak epistemic game model to satisfy the ex interim condition (ExIC) and the adequate valuation condition (AVC). That is, $M$ is a weak epistemic game model in which the formula $\bigwedge_{\psi \in \Delta_{0}} \psi$ is true (i.e., $M, w \vDash \bigwedge_{\psi \in \Delta_{0}} \psi$ for all $w$ in $M$ ) iff $M$ is an epistemic game model. Therefore, the class WEGM in which the formula $\bigwedge_{\psi \in \Delta_{0}} \psi$ is true coincides with the class EGM.

The following Proposition 6 highlights that, thanks to the universal modality $\square$, the logical consequence problem relative to the class WEGM can be reduced to the validity problem relative to the class WEGM. The proof of the proposition is trivial, as we just need to apply the definitions of validity and logical consequence relative to WEGM.

Proposition 6 For every EDGL-formula $\varphi$ and for every finite set $\triangle$ of EDGLformulas, $\Delta \vDash_{W E G M} \varphi$ iff $\vDash_{W E G M} \square \bigwedge_{\psi \in \Delta} \psi \rightarrow \varphi$.

The following Lemma 2 provides an axiomatization result for EDGL relative to the class WEGM.

Lemma 2 The set of validities of the logic EDGL relative to the class WEGM is completely axiomatized by the groups of axioms (1),(2),(3),(4) and (5) and by the rules of inference (7) and (8) in Fig. 5.

Proof The proof is divided into three steps.

Step 1 The first step consists in providing an alternative semantics for EDGL relative to the class of enriched weak epistemic game models (EWEGM). An enriched weak epistemic game model is a tuple $M=\left\langle W, \sim_{1}, \ldots, \sim_{n}, \rightarrow_{1}, \ldots, \rightarrow_{n}, \sim, v\right\rangle$ where $\left\langle W, \sim_{1}, \ldots, \sim_{n}, \rightarrow_{1}, \ldots, \rightarrow_{n}, v\right\rangle$ is a weak epistemic game model and $\sim$ is an equivalence relation on $W$ such that:

(C1) for all $i \in N, \sim_{i} \subseteq \sim$;

(C2) for all $u_{1}, \ldots, u_{n} \in W$ : if $u_{i} \sim u_{j}$ for all $i, j \in\{1, \ldots, n\}$ then $\sim_{1}\left(u_{1}\right) \cap$ $\ldots \cap \sim_{n}\left(u_{n}\right) \neq \emptyset$.

The truth conditions of EDGL formulas relative to the class EWEGM are exactly like the truth conditions of EDGL formulas relative to the classes WEGM and EGM, except for the universal modality $\square$ that is interpreted as follows. Let $M=\left\langle W, \sim_{1}\right.$ $\left., \ldots, \sim_{n}, \rightarrow_{1}, \ldots, \rightarrow_{n}, \sim, v\right\rangle$ be a EWEGM and let $w$ be a world in $M$. Then: 
- $M, w \vDash \square \varphi$ iff $\forall w^{\prime}$ such that $w \sim w^{\prime}, M, w^{\prime} \vDash \varphi$

Step 2 The second step consists in proving that the set of validities of EDGL relative to the class EWEGM is completely axiomatized by the groups of axioms (1), (2), (3), (4) and (5) and by the rules of inference (7) and (8) in Fig. 5.

It is a routine task to check that all principles in Fig. 5 except Axiom (5d) correspond one-to-one to their semantic counterparts on the models in the class EWEGM. This can be easily checked by using the existing algorithm SQEMA (Conradie et al. 2006): for every axiom in Fig. 5, it allows us to compute the corresponding first-order condition on the models in the class EWEGM.

In particular, the group of axioms (2) together with the inference rule (8) correspond to the fact that $\sim$ is an equivalence relations. ${ }^{3}$ The group of axioms (3) corresponds to the fact that $\sim_{i}$ is an equivalence relation, while Axiom (4b) corresponds to the seriality of the relation $\rightarrow_{i}$.

Remark 1 Note that the necessitation rules for the knowledge modality (i.e., from $\varphi$ infer $K_{i} \varphi$ ) and for the belief modality (i.e., from $\varphi$ infer $B_{i} \varphi$ ) do not need to be added to the axiomatization, as they are provable by Axioms (5a), Axiom (5b), the inference rule (7) and the inference rule (8). ${ }^{4}$

As to the group of axioms (5) we have the following correspondences: Axiom (5a) corresponds to the preceding condition $\mathrm{C} 1: \sim_{i} \subseteq \sim$; Axiom (5b) corresponds to the following condition in the definition of epistemic game model: $\rightarrow \rightarrow_{i} \subseteq \sim_{i}$; Axiom (5c) corresponds to the following condition: for all $w, v \in W$, if $w \sim_{i} v$ then $\rightarrow_{i}(v) \subseteq \rightarrow_{i}$ $(w)$. Because of the reflexivity of the relation $\sim_{i}$ the latter is equivalent to the following condition in the definition of epistemic game model: for all $w, v \in W$, if $w \sim_{i} v$ then $\rightarrow_{i}(v)=\rightarrow_{i}(w)$.

As to Axiom (5d) a bit more work is required. First of all, it is a routine task to verify that, in terms of correspondence theory, Axiom (5d) corresponds to the following condition:

$\left(\mathrm{C} 2^{*}\right)$ for all $w, u_{1}, \ldots, u_{n} \in W$ : if $u_{1}, \ldots, u_{n} \in \sim(w)$ then there is $v \in W$ such that $v \in \sim(w)$ and $\sim_{i}(v) \subseteq \sim_{i}\left(u_{i}\right)$ for all $i \in N$

Secondly, one can prove that the condition $\mathrm{C} 2 *$ and the condition $\mathrm{C} 2$ are equivalent. Let us prove first that C2 implies C2*. Suppose that $u_{1}, \ldots, u_{n} \in \sim(w)$. Hence, by condition $\mathrm{C} 2$ and the fact that $\sim$ is an equivalence relation, $\sim(w) \cap \sim_{1}\left(u_{1}\right) \cap$ $\ldots \cap \sim_{n}\left(u_{n}\right) \neq \varnothing$. It follows that there exists $v \in W$ such that $v \in \sim(w), v \in \sim_{1}$ $\left(u_{1}\right), \ldots, v \in \sim_{n}\left(u_{n}\right)$. Since every $\sim_{i}$ is an equivalence relation, for every $i \in N$, if $v \in \sim_{i}\left(u_{i}\right)$ then $\sim_{i}(v)=\sim_{i}\left(u_{i}\right)$. Thus, we can conclude that there exists $v \in W$ such that $v \in \sim(w)$ and $\sim_{i}(v) \subseteq \sim_{i}\left(u_{i}\right)$ for all $i \in N$.

Now let us prove that $\mathrm{C} 2 *$ implies C2. Suppose that $u_{i} \sim u_{j}$ for all $i, j \in\{1, \ldots, n\}$. It follows that $u_{1}, \ldots, u_{n} \in \sim(w)$ for some $w$. Hence, by condition $\mathrm{C} 2 *$, there are

\footnotetext{
${ }^{3}$ Specifically, Axiom (2b) corresponds to reflexivity of the relation $\sim$, Axiom (2c) to transitivity and Axiom (2d) to symmetry.

4 Suppose $\varphi$. Hence, by the inference rule (8), we infer $\square \varphi$. Thus, by Axiom (5a) and the inference rule (7), we infer $K_{i} \varphi$. Furthermore, by Axiom (5b) and the inference rule (7), we infer $B_{i} \varphi$.
} 
$w, v \in W$ such that $v \in \sim(w)$ and $\sim_{i}(v) \subseteq \sim_{i}\left(u_{i}\right)$ for all $i \in N$. Since every $\sim_{i}$ is an equivalence relation, for every $i \in N$, if $\sim_{i}(v) \subseteq \sim_{i}\left(u_{i}\right)$ then $\sim_{i}(v)=\sim_{i}\left(u_{i}\right)$. Thus, we can conclude that there are $w, v \in W$ such that $v \in \sim(w)$ and $\sim_{i}(v)=\sim_{i}\left(u_{i}\right)$ for all $i \in N$. By the fact that every relation $\sim_{i}$ is reflexive, it follows that there are $w, v \in W$ such that $v \in \sim(w)$ and $v \in \sim_{i}\left(u_{i}\right)$ for all $i \in N$. Hence, $\sim_{1}\left(u_{1}\right) \cap \ldots \cap \sim_{n}\left(u_{n}\right) \neq \emptyset$.

It is routine, too, to check that all principles given in Fig. 5 are in the so-called Sahlqvist class (Sahlqvist 1975). Thus, because of the general Sahlqvist completeness theorem (cf. Blackburn et al. 2001, Theorem 3.54), they are complete with respect to the defined model classes.

Step 3 The third step consists in proving that the EDGL-semantics relative to the class EWEGM and the EDGL-semantics relative to the class WEGM are equivalent. Specifically, we show that for every EDGL-formula formula $\varphi, \varphi$ is satisfiable in the class WEGM iff $\varphi$ is satisfiable in the class EWEGM.

$(\Rightarrow)$ Let us prove the left-to-right direction. Suppose $\varphi$ is satisfiable in the class WEGM. This means that there is a weak epistemic game model $M=\left\langle W, \sim_{1}, \ldots, \sim_{n}\right.$ $\left., \rightarrow_{1}, \ldots, \rightarrow_{n}, v\right\rangle$ and a world $w \in W$ such that $M, w \vDash \varphi$. We can build a corresponding enriched weak epistemic game model $M^{\prime}=\left\langle W, \sim_{1}, \ldots, \sim_{n}, \rightarrow_{1}, \ldots, \rightarrow_{n}\right.$ $, \sim, v\rangle$ with $\sim=W \times W$. Clearly, $M^{\prime}, w \vDash \varphi$.

$(\Leftarrow)$ Let us prove the right-to-left direction. Suppose $\varphi$ is satisfiable in the class EWEGM. This means that there is an enriched weak epistemic game model $M=\left\langle W, \sim_{1}, \ldots, \sim_{n}, \rightarrow_{1}, \ldots, \rightarrow_{n}, \sim, v\right\rangle$ and a world $w \in W$ such that $M, w \vDash \varphi$. We can now build a weak epistemic game model $M^{\prime}=\left\langle W^{\prime}, \sim_{1}^{\prime}, \ldots, \sim_{n}^{\prime}, \rightarrow_{1}^{\prime}\right.$ $\left., \ldots, \rightarrow_{n}^{\prime}, v^{\prime}\right\rangle$ that corresponds to $M$. The construction of $M^{\prime}$ is made in two steps. We first consider the submodel $M_{w}=\left\langle W_{w}, \sim_{1, w}, \ldots, \sim_{n, w}, \rightarrow_{1, w}, \ldots, \rightarrow_{n, w}, \sim_{w}, v_{w}\right\rangle$ generated from $M$ and $w$ (cf. Blackburn et al. 2001, Definition 2.5): by the generated submodel property (cf. Blackburn et al. 2001, Proposition 2.6) we have $M_{w}, w \vDash \varphi$. $M_{w}$ is also an enriched weak epistemic game model, and $\sim_{w}=W_{w} \times W_{w}$. The latter means that the operator $\square$ is interpreted as a universal modal operator. Finally, we can define $M^{\prime}=\left\langle W^{\prime}, \sim_{1}^{\prime}, \ldots, \sim_{n}^{\prime}, \rightarrow_{1}^{\prime}, \ldots, \rightarrow_{n}^{\prime}, v^{\prime}\right\rangle$ as follows:

$-W^{\prime}=W_{w}$;

- for every $i \in N, \sim_{i}^{\prime}=\sim_{i, w}$ and $\rightarrow_{i}^{\prime}=\rightarrow_{i, w}$;

$-v^{\prime}=v_{w}$.

It is a routine task to check that $M^{\prime}$ is indeed a weak epistemic game model and, by induction on the structure of $\varphi$, that we have $M^{\prime}, w \vDash \varphi$.

Lemma 2 is a consequence of: (i) the equivalence between the EDGL-semantics relative to the class EWEGM and the EDGL-semantics relative to the class WEGM (proved in Step 3) and, (ii) the completeness result for EDGL relative to the class EWEGM (proved in Step 2).

The last element we need for proving Theorem 5 is the following Proposition 7. Let $\vdash_{E D G L} \varphi$ and $\| \vdash_{E D G L} \varphi$ mean, respectively, that the EDGL-formula $\varphi$ is provable via the groups of axioms (1),(2),(3),(4), (5) and (6) and the rules of inference (7) and (8) in Fig. 5 and that the EDGL-formula $\varphi$ is provable via the groups of axioms (1),(2),(3),(4) and (5) and the rules of inference (7) and (8) in Fig. 5.

Proposition 7 For every EDGL-formula $\varphi$, if $\| \vdash_{E D G L} \square \bigwedge_{\psi \in \Delta_{0}} \psi \rightarrow \varphi$ then $\vdash_{E D G L} \varphi$, where $\Delta_{0}$ is defined as in Proposition 5. 
Proof Suppose $\| \vdash_{E D G L} \square \bigwedge_{\psi \in \Delta_{0}} \psi \rightarrow \varphi$. Hence, $\vdash_{E D G L} \square \bigwedge_{\psi \in \Delta_{0}} \psi \rightarrow \varphi$.

By the inference rule (8) (viz. necessitation for $\square$ ) and the group of axioms (6), we have $\vdash_{E D G L} \bigwedge_{\psi \in \Delta_{0}} \square \psi$. By Axiom 2(a), we can derive $\vdash_{E D G L} \square \bigwedge_{\psi \in \Delta_{0}} \psi$. Consequently, by the inference rule (7) (viz. modus ponens), we have that $\vdash_{E D G L} \varphi$.

Propositions 5, 6 and 7 together with Lemma 2 are sufficient to prove Theorem 5.

Suppose that $\vDash_{E G M} \varphi$. Hence, by Propositions 5 and $6, \vDash_{W E G M} \square \bigwedge_{\psi \in \Delta_{0}} \psi \rightarrow \varphi$. By Lemma 2, it follows that $\| \vdash_{E D G L} \square \bigwedge_{\psi \in \Delta_{0}} \psi \rightarrow \varphi$. Hence, by Proposition 7, $\vdash_{E D G L} \varphi$.

As to the logic DGL, namely the fragment of the logic EDGL presented in Sect. 2, we have the following axiomatization result. We do not prove it here, as the proof follows the general lines of the proof of Theorem 5 .

Theorem 6 The set of validities of the logic DGL relative to the class of doxastic game models (DGM) is completely axiomatized by the groups of axioms (1), (4) and (6) and the inference rule (7) in Fig. 5, plus the following axioms and rules of inference for the belief modality $B_{i}$ :

- $B_{i} \varphi \rightarrow B_{i} B_{i} \varphi$

$-\widehat{B}_{i} \varphi \rightarrow B_{i} \widehat{B}_{i} \varphi$

- From $\varphi$ infer $B_{i} \varphi$

Note that differently from EDGL, Axioms 4 and 5 as well as the necessitation rule for the belief modality $B_{i}$ must be added to the axiomatics, as they are not derivable from the other principles.

\section{Bridge between EDGL and STIT}

In this section we propose a bridge between what we have done so far and one of the most prominent logics of action, namely STIT logic (Belnap et al. 2001; Horty 2001). Specifically, we show that the logic EDGL can be linearly embedded into atemporal individual STIT. More generally, the significance of this result lies in the possibility of building a connection between the area of epistemic logics and the area of logics of action.

\subsection{Atemporal individual STIT}

STIT logic (the logic of seeing to it that) by Belnap et al. (2001) is the logic of sentences of the form "agent $i$ sees to it that $\varphi$ is true". In Horty (2001) Horty extends Belnap et al.'s STIT framework by operators of group agency in order to express sentences of the form "the group of agents $G$ sees to it that $\varphi$ is true". One might use the terms 'individual STIT' and 'group STIT' to designate, respectively, Belnap et al.'s STIT logic, in which only agency operators for the individuals are given, and Horty's variant of STIT, with both agency operators for the individuals and agency operators for the groups. An important distinction in the family of STIT languages is between 
'temporal STIT' (i.e., STIT with tense operators in the object language) and 'atemporal STIT' (i.e., STIT without tense operators). A Kripke semantics for atemporal individual STIT is provided in Balbiani et al. (2008), Herzig and Schwarzentruber (2008) and Lorini and Schwarzentruber (2011), while an extension of this semantics to atemporal group STIT is provided in Herzig and Schwarzentruber (2008) and Lorini and Schwarzentruber (2011)

Here, we build a bridge between EDGL and atemporal individual STIT.

First let us recall the syntax and the semantics of atemporal individual STIT (STIT, for short). The language $\mathscr{L}_{\text {STIT }}(\mathbf{P}, A G T)$ of this logic is built from an infinite set of atomic formulas $\mathbf{P}$ and a finite set of agents $A G T=\{1, \ldots, n\}$ and is defined by the following grammar:

$$
\varphi::=p|\neg \varphi| \varphi \wedge \varphi|[i] \varphi| \square \varphi
$$

where $p \in \mathbf{P}$ and $i \in A G T$. The construction $[i] \varphi$ is read "agent $i$ sees to it that $\varphi$ is true regardless of what the other agents choose". We define the dual of $[i]$ as follows: $\langle i\rangle \varphi:=\neg[i] \neg \varphi$. $\square$ is the universal modality and $\square \varphi$ is read " $\varphi$ is true regardless of what every agent chooses" or simply " $\varphi$ is necessarily true".

Definition 6 (STIT-Kripke model Balbiani et al. 2008; Herzig and Schwarzentruber 2008) A STIT-Kripke model $S M=\left\langle W, R_{1}, \ldots, R_{n}, V\right\rangle$ is a tuple where:

- $W$ is a countable set of possible worlds;

$-R_{1}, \ldots, R_{n}$ are equivalence relations on $W$ that satisfies the following independence of choices condition (ICC):

- $R_{1}\left(w_{1}\right) \cap \ldots \cap R_{n}\left(w_{n}\right) \neq \emptyset$, for every $w_{1}, \ldots, w_{n} \in W$

$-V: W \longrightarrow \mathscr{P}(\mathbf{P})$ is a valuation function for atomic propositions;

with $R_{i}(w)=\left\{w^{\prime}: w R_{i} w^{\prime}\right\}$.

The partition induced by the equivalence relation $R_{i}$ is the set of possible choices of the agent $i$. Indeed, in STIT a choice of an agent $i$ at a given world $w$ is identified with the set of possible worlds $R_{i}(w)$. We call $R_{i}(w)$ the set of possible outcomes of agent $i$ 's choice at world $w$, in the sense that agent $i$ 's current choice at $w$ forces the possible worlds to be in $R_{i}(w)$. The independence of choices condition (ICC) states that, whatever each agent decides to do, the set of outcomes corresponding to the joint action of all agents is non-empty. More intuitively, this means that agents can never be deprived of choices due to the choices made by other agents.

Example 1 The tuple $S M=\left\langle W, R_{1}, R_{2}, V\right\rangle$ defined by:

$-W=\{w, u, v, r, s, t, z\}$

$-R_{1}=\{w, u, v\}^{2} \cup\{r, s\}^{2} \cup\{t, z\}^{2}$;

$-R_{2}=\{w, r, t\}^{2} \cup\{u, v, s, z\}^{2}$;

- for all $p \in \mathbf{P}, V(p)=\emptyset$.

is a STIT-Kripke model. Figure 6 shows the model SM. The equivalence classes induced by the equivalence relation $R_{1}$ are represented by ellipses and correspond to the choices of agent 1 . The equivalence classes induced by the equivalence relation $R_{2}$ 
Fig. 6 The STIT-model SM

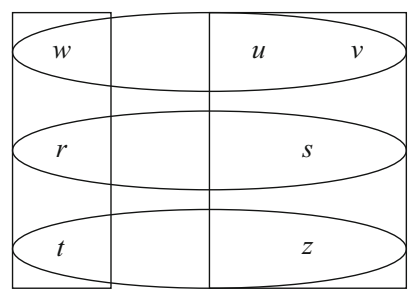

are represented by rectangles and correspond to the choices of agent 2. For example, the choice of agent 1 at world $u$ is $\{w, u, v\}$ whereas the choice of agent 2 at world $u$ is $\{u, v, s, z\}$. Note that independence of choices condition (ICC) ensures that for any choice of agent 1 and for any choice of agent 2 the intersection between these two choices is non-empty. That is, for any equivalence class induced by the relation $R_{1}$ and for any equivalence class induced by the relation $R_{2}$, the intersection between these two equivalence classes is non-empty.

Given a STIT-Kripke model $S M=\left\langle W, R_{1}, \ldots, R_{n}, V\right\rangle$ and a world $w$ in $S M$, the truth conditions of STIT formulas are the following:

- SM, $w \vDash p$ iff $p \in V(w)$

$-S M, w \vDash \neg \varphi$ iff $S M, w \not \models \varphi$

- $S M, w \vDash \varphi \wedge \psi$ iff $S M, w \vDash \varphi$ and $S M, w \vDash \psi$

- $S M, w \vDash[i] \varphi$ iff $\forall w^{\prime}$ s.t. $w R_{i} w^{\prime}, S M, v \vDash \varphi$

- $S M, w \vDash \square \varphi$ iff $\forall w^{\prime} \in W, S M, v \vDash \varphi$

We say that a formula $\varphi$ of the language $\mathscr{L}_{S T I T}(\mathbf{P}, A G T)$ is true in a STIT-Kripke model $S M=\left\langle W, R_{1}, \ldots, R_{n}, V\right\rangle$ if $\forall w \in W$ we have $M, w \vDash \varphi$. Then, $\varphi$ is STITvalid, denoted by $\vDash_{S T I T} \varphi$, if $\varphi$ is true in $S M$ for all STIT-Kripke models $S M$. Finally, $\varphi$ is STIT-satisfiable if $\neg \varphi$ is not STIT-valid.

\subsection{Truth-preserving embedding of EDGL into STIT}

We define the following linear translation $\operatorname{tr}$ from $\mathscr{L}_{E D G L}(\mathbf{G})$ to $\mathscr{L}_{S T I T}(\mathbf{P}, N)$ where $\mathbf{P}$ is a proper extension of $\Phi$ (i.e., $\Phi \subset \mathbf{P}$ ) and $x_{i}$ is a special atoms in $\mathbf{P} \backslash \Phi$ that allows to identify the worlds in agent $i$ 's belief set (i.e., the worlds in $i$ 's information set with probability higher than 0 ):

$$
\begin{aligned}
\operatorname{tr}\left(p l\left(a_{i}\right)\right) & =\operatorname{pl}\left(a_{i}\right) \text { for } p l\left(a_{i}\right) \in \Phi \\
\operatorname{tr}(\neg \varphi) & =\neg \operatorname{tr}(\varphi) \\
\operatorname{tr}(\varphi \wedge \psi) & =\operatorname{tr}(\varphi) \wedge \operatorname{tr}(\psi) \\
\operatorname{tr}\left(K_{i} \varphi\right) & =[i] \operatorname{tr}(\varphi) \\
\operatorname{tr}\left(B_{i} \varphi\right) & =[i]\left(x_{i} \rightarrow \operatorname{tr}(\varphi)\right) \\
\operatorname{tr}(\square \varphi) & =\square \operatorname{tr}(\varphi)
\end{aligned}
$$

In order to provide an embedding of EDGL into STIT, we need to define the following STIT formulas which capture four basic assumptions of EDGL: 


$$
\begin{aligned}
\text { OneAct }: & =\square\left(\bigwedge_{i \in N} \bigvee_{a_{i} \in A_{i}} p l\left(a_{i}\right)\right) \\
\text { NoMoreAct }: & =\square\left(\bigwedge_{i \in N} \bigwedge_{a_{i} \neq a_{i}^{\prime}}\left(p l\left(a_{i}\right) \rightarrow \neg p l\left(a_{i}^{\prime}\right)\right)\right) \\
\text { ExInt }: & =\square\left(\bigwedge_{i \in N} \bigwedge_{a_{i} \in A_{i}}\left(p l\left(a_{i}\right) \rightarrow[i] p l\left(a_{i}\right)\right)\right) \\
\text { OneProb }: & =\square\left(\bigwedge_{i \in N}\langle i\rangle x_{i}\right)
\end{aligned}
$$

Formula OneAct forces the valuation function to assign at least one action to each player in each world. Formula NoMoreAct forces the valuation function to assign no more than one action to each player in each world. Formula ExInt correspond to the ex interim condition. Finally, formula OneProb forces every player to have at least one world in his belief set.

The following theorem highlights that the preceding translation provides a correct embedding of the logic EDGL into the logic STIT.

Theorem 7 A formula $\varphi$ of the language $\mathscr{L}_{E D G L}(\boldsymbol{G})$ is satisfiable relative to the class of epistemic game models $(\boldsymbol{E G M})$ iff OneAct $\wedge$ NoMoreAct $\wedge$ ExInt $\wedge$ OneProb $\wedge \operatorname{tr}(\varphi)$ is STIT-satisfiable.

Proof We first prove the left-to-right direction of the theorem. Let $\varphi$ be satisfiable relative to the class of epistemic game models. This means that there exists an epistemic game model $M=\left\langle W, \sim_{1}, \ldots, \sim_{n}, \rightarrow_{1}, \ldots, \rightarrow_{n}, v\right\rangle$ and a world $w \in W$ such that $M, w \vDash \varphi$. We define a corresponding structure $S M=\left\langle W, R_{1}, \ldots, R_{n}, V\right\rangle$ such that: (i) $R_{i}=\sim_{i}$ for all $i \in N$, (ii) for all $p l\left(a_{i}\right) \in \Phi$ and for all $w \in W, p l\left(a_{i}\right) \in V(w)$ iff $p l\left(a_{i}\right) \in v(w)$, (iii) for all $i \in N$ and for all $w \in W, x_{i} \in V(w)$ iff $w \in \rightarrow_{i}(w)$, and (iv) for all $p \in \mathbf{P} \backslash\left(\Phi \cup\left\{x_{1}, \ldots, x_{n}\right\}\right),\|p\|^{S M}=\{w \in W: p \in V(w)\}=\emptyset$. It is easy to check that $S M$ is a STIT-Kripke model and that $S M, w \vDash$ OneAct $\wedge$ NoMoreAct $\wedge$ ExInt $\wedge$ OneProb. Moreover, by induction on the structure of $\varphi$, we can prove that $S M, w \vDash \operatorname{tr}(\varphi)$. We prove only some cases. The induction basis is clear. Let us prove the inductive case $\varphi=B_{i} \psi$. Suppose $M, w \vDash B_{i} \psi$. This means that $M, w^{\prime} \vDash \psi$ for all $w^{\prime} \in \rightarrow_{i}(w)$. Thus, by inductive hypothesis, $S M, w^{\prime} \vDash \operatorname{tr}(\psi)$ for all $w^{\prime} \in R_{i}(w)$ such that $x_{i} \in V\left(w^{\prime}\right)$. The latter is equivalent to $S M, w^{\prime} \vDash[i]\left(x_{i} \rightarrow \operatorname{tr}(\psi)\right)$.

Now, let us prove right-to-left direction. Let OneAct $\wedge$ NoMoreAct $\wedge$ ExInt $\wedge$ OneProb $\wedge \operatorname{tr}(\varphi)$ be STIT-satisfiable. This means that there exists a STIT-Kripke model $S M=\left\langle W, R_{1}, \ldots, R_{n}, V\right\rangle$ and a world $w$ in $S M$ such that $S M, w \vDash$ OneAct $\wedge$ NoMoreAct $\wedge$ ExInt $\wedge$ OneProb $\wedge \operatorname{tr}(\varphi)$. We define a corresponding structure $M=\left\langle W, \sim_{1}, \ldots, \sim_{n}, \rightarrow_{1}, \ldots, \rightarrow_{n}, v\right\rangle$ such that: (i) $\sim_{i}=R_{i}$ for all $i \in N$, (ii) for all $i \in N, \rightarrow_{i}=\left\{\left(w, w^{\prime}\right): w R_{i} w^{\prime}\right.$ and $\left.M, w^{\prime} \vDash x_{i}\right\}$, (iii) for all $p l\left(a_{i}\right) \in \Phi$ and for all $w \in W, p l\left(a_{i}\right) \in V(w)$ iff $p l\left(a_{i}\right) \in v(w)$. It is easy to check that $M$ is an epistemic game model, because $S M, w \vDash$ OneAct $\wedge$ NoMoreAct $\wedge$ ExInt $\wedge$ OneProb. Moreover, by induction on the structure of $\varphi$, we can prove that $M, w \vDash \varphi$. We prove only some cases. The induction basis is clear. Let us prove the inductive case $\varphi=B_{i} \psi$. Suppose $S M, w \vDash$ OneAct $\wedge$ NoMoreAct $\wedge$ ExInt $\wedge$ OneProb $\wedge \operatorname{tr}\left(B_{i} \psi\right)$. The latter 
implies that $S M, w \vDash[i]\left(x_{i} \rightarrow \operatorname{tr}(\psi)\right)$. Thus, for all $w^{\prime} \in R_{i}(w)$ if $S M, w^{\prime} \vDash x_{i}$ then $S M, w^{\prime} \vDash \operatorname{tr}(\psi)$. Hence, by inductive hypothesis, $M, w^{\prime} \models \psi$ for all $w^{\prime} \in \rightarrow_{i}(w)$. Thus, $M, w \models B_{i} \psi$.

\section{Probabilistic extension}

In this section we want to show how to extend our analysis about the equivalence between Kripke-style semantics and type space semantics for epistemic modal languages to a modal language with probabilistic beliefs $\mathscr{L}_{E P G L}(\mathbf{G})$, interpreted over a specific class of Kripke models called epistemic-probabilistic game models (EPGM). The resulting logic EPGL (epistemic-probabilistic game logic) will then be an extension of the logic EDGL, that was itself introduced as an extension of DGL. Consequently, this section follows an approach that is incremental with respect to the logics that have been introduced so far.

\subsection{Language}

Let us first introduce a probabilistic language $\mathscr{L}_{E P G L}(\mathbf{G})$ for epistemic-probabilistic game logic EPGL, defined by the grammar:

$$
\varphi::=p l\left(a_{i}\right)|\neg \varphi| \varphi \wedge \varphi\left|K_{i} \varphi\right| P_{i}^{r} \varphi \mid \square \varphi
$$

where $a_{i} \in A_{i}, i \in N$ and $r \in \mathbb{Q}$.

The language $\mathscr{L}_{E P G L}(\mathbf{G})$ is the language $\mathscr{L}_{E D G L}(\mathbf{G})$ of EDGL introduced in Sect. 3 with the difference of replacing the qualitative belief operator $B_{i}$ with the probabilistic belief operator $P_{i}^{r}$, where $P_{i}^{r} \varphi$ has to be read "player $i$ believes with probability at least $r$ that $\varphi$ is true". It is easy to see that this language is incremental relative to $\mathscr{L}_{E D G L}(\mathbf{G})$ : the belief operator $B_{i}$ of $\mathscr{L}_{E D G L}(\mathbf{G})$ is expressible in $\mathscr{L}_{E P G L}(\mathbf{G})$ by the operator $P_{i}^{1}$.

\subsection{Semantics}

\subsubsection{Type spaces}

The interpretation of the new formulas $P_{i}^{r} \varphi$ in a type space $\mathscr{T}$ is as one could expect, and it is given by the following clause:

- $\mathscr{T}, t \vDash P_{i}^{r} \varphi$ iff $\forall t^{\prime} \in T$ if $t_{i}^{\prime}=t_{i}$ then $\sum_{t_{-i}^{\prime}: \mathscr{T}, t_{i}^{\prime} \vDash \varphi} \beta_{i}\left(t_{i}\right)\left(t_{-i}^{\prime}\right) \geq r$.

All the other clauses are the same as in Sect. 3. As mentioned before, when $r=1$ we can show the equivalence with respect to the clause for $B_{i}$. Indeed,

$$
\mathscr{T}, t \vDash B_{i} \varphi
$$


iff

$$
\forall t^{\prime} \in T \text { if } t_{i}^{\prime}=t_{i} \text { and } \beta_{i}\left(t_{i}\right)\left(t_{-i}^{\prime}\right)>0 \text { then } \mathscr{T}, t^{\prime} \vDash \varphi
$$

iff

$$
\forall t^{\prime} \in T \text { if } t_{i}^{\prime}=t_{i} \text { then } \sum_{t_{-i}^{\prime}: \mathscr{T}, t_{i}^{\prime} \vDash \varphi} \beta_{i}\left(t_{i}\right)\left(t_{-i}^{\prime}\right)=1
$$

iff

$$
\mathscr{T}, t \vDash P_{i}^{1} \varphi \text {. }
$$

\subsubsection{Epistemic-probabilistic models}

When we come to consider how to interpret the language $\mathscr{L}_{E P G L}(\mathbf{G})$ over Kripke models we firstly need to endow the structure with probabilistic belief relations for the agents. The resulting Kripke model will be an epistemic-probabilistic model, formally defined as follows.

Definition 7 (Epistemic-probabilistic model) An epistemic-probabilistic model is a tuple $M=\left\langle W, \sim_{1}, \ldots, \sim_{n}, \theta_{1}, \ldots, \theta_{n}, v\right\rangle$ where $W, \sim_{i}$ and $v$ are defined as in Definition 4 , and $\theta_{i}$ is a function mapping every world $w \in W$ to a probability distribution $\theta_{i, w}$ over the worlds in $\sim_{i}(w)$ and satisfying the following conditions:

$-\sum_{w^{\prime} \in \sim_{i}(w)} \theta_{i, w}\left(w^{\prime}\right)=1$;

- if $w \sim_{i} w^{\prime}$ then $\theta_{i, w}=\theta_{i, w^{\prime}}$.

In order to interpret the language $\mathscr{L}_{E P G L}(\mathbf{G})$ on epistemic-probabilistic models we want to identify a subclass of them that we call epistemic-probabilistic game models EPGM.

Definition 8 (Epistemic-probabilistic game model) An epistemic-probabilistic game model EPGM is an epistemic-probabilistic model that satisfies conditions AVC and EIC of Definitions 2 and 5, plus the following condition ExIC':

- Ex interim condition revisited (ExIC'):

$$
\begin{aligned}
& \forall i \in N, \forall w, w^{\prime} \in W, \forall a_{i} \in A_{i}, \text { if } w \sim_{i} w^{\prime} \text { and } p l\left(a_{i}\right) \in \\
& v(w) \text { then } p l\left(a_{i}\right) \in v\left(w^{\prime}\right) .
\end{aligned}
$$

Now we can give the conditions to interpret the language $\mathscr{L}_{E P G L}(\mathbf{G})$ over epistemic-probabilistic game models EPGM. Apart from $P_{i}^{r}$-formulas, the truth conditions for all the other formulas remain the same as for EDGM. The clause for $P_{i}^{r}$-formulas is as follows:

$-M, w \vDash P_{i}^{r} \varphi$ iff $\sum_{w^{\prime} \in \sim_{i}(w): M, w^{\prime} \vDash \varphi} \theta_{i, w}\left(w^{\prime}\right) \geq r$. 


\subsection{Correspondence}

It is also possible to establish correspondence results between type spaces and epistemic-probabilistic game models relative to the language $\mathscr{L}_{E P G L}(\mathbf{G})$. Given an arbitrary type space $\mathscr{T}$, the corresponding epistemic-probabilistic model $M^{\mathscr{T}}$ is defined as the tuple $M^{\mathscr{T}}=\left\langle W,\left(\sim_{i}\right)_{i \in N},\left(\theta_{i, w}\right)_{i \in N, w \in W}, v\right\rangle$ where:

$-W=T$ is the set of worlds;

$-v: W \longrightarrow \mathscr{P}(\Phi)$ is the valuation function such that $p l\left(a_{i}\right) \in v(w)$ iff $\sigma_{i}\left(t_{i}\right)=a_{i}$;

$-\sim_{i}$ is the accessibility relation of player $i$, given by: $\forall w, w^{\prime} \in W, w \sim_{i} w^{\prime}$ iff $t_{i}=$ $t_{i}^{\prime}$

- $\theta_{i, w}$ is the probabilistic belief relation of player $i$ at world $w$, defined as follows: $\theta_{i, w}\left(w^{\prime}\right)=r$ iff $\beta_{i}\left(t_{i}\right)\left(t_{-i}^{\prime}\right)=r$ and $t_{i}=t_{i}^{\prime}$.

It is easy to see that the Kripke model that we get after the transformation is an epistemic-probabilistic game model.

Proposition $8 M^{\mathscr{T}}$ is an epistemic-probabilistic game model.

Proof AVC and EIC conditions hold unchanged with respect to EGM. The only thing that differs from epistemic game models is ExIC'. Then, ExIC' also holds in $M^{\mathscr{T}}$ since each type is associated with one and only one action and two worlds $w$ and $w^{\prime}$ belong to the same partition cell of player $i$ if and only if the type of $i$ at $w$ is the same as $i$ 's type at $w^{\prime}$.

As for DGM and EGM before, we conclude the first half of the proof with a theorem showing that if a formula of $\mathscr{L}_{E P G L}(\mathbf{G})$ is satisfiable in a type space $\mathscr{T}$, then it is also satisfiable in the corresponding EPGM $M^{\mathscr{T}}$.

Theorem 8 Let $t \in T$ be a state in $\mathscr{T}$ and let $w \in W$ be the corresponding world in $M^{\mathscr{T}}$. For any $\varphi$ in $\mathscr{L}_{E P G L}(\boldsymbol{G})$, if $\mathscr{T}, t \vDash \varphi$ then $M^{\mathscr{T}}, w \vDash \varphi$.

Proof By induction on the structure of $\varphi$. We prove just the case $\varphi=P_{i}^{r} \psi$ since the others are similar to Sect. 3. Suppose $\mathscr{T}, t \vDash P_{i}^{r} \psi$. Then, $\sum_{t_{-i}^{\prime}: \mathscr{T}, t_{i}^{\prime} \vDash \psi} \beta_{i}\left(t_{i}\right)\left(t_{-i}^{\prime}\right) \geq r$. By inductive hypothesis, $\sum_{w^{\prime} \in \sim_{i}(w): M, w^{\prime} \vDash \psi} \theta_{i, w}\left(w^{\prime}\right) \geq r$, hence $M^{\mathscr{T}}, w \vDash P_{i}^{r} \psi$.

In the second half of the proof the other direction is carried out: we show that given any epistemic-probabilistic game model $M$ it is possible to associate with it the corresponding type space $\mathscr{T}^{M}$, and that $M$ and $\mathscr{T}^{M}$ are semantically equivalent with respect to $\mathscr{L}_{E P G L}(\mathbf{G})$.

Apart from the probabilistic belief relation $\theta_{i, w}$, the construction of the type space $\mathscr{T}^{M}$ is the same as for the case of epistemic game models. What we need to define here is the belief function $\beta_{i}$ for player $i$, given the probabilistic belief relation $\theta_{i, w}$ of player $i$ in $M$. Then, for an arbitrary $M=\left\langle W,\left(\sim_{i}\right)_{i \in N},\left(\theta_{i, w}\right)_{i \in N, w \in W}, v\right\rangle$, we can associate with it the corresponding type space $\mathscr{T}^{M}=\left\langle T_{1}, \ldots, T_{n}, \beta_{1}, \ldots, \beta_{n}, \sigma_{1}, \ldots, \sigma_{n}\right\rangle$ defined as follows:

- a type $t_{i}$ of player $i$ for each $i$ 's partition cell $\sim_{i}(w)$ in $M: T_{i}=\left\{\sim_{i}(w): w \in W\right\}$;

- an action $a_{i}$ of $t_{i}$ specified by $v: \sigma_{i}\left(t_{i}\right)=a_{i}$ iff $p l\left(a_{i}\right) \in v(w)$; 
- a belief function $\beta_{i}$ such that $\beta_{i}\left(t_{i}\right)\left(t_{-i}^{\prime}\right)=r$ iff $\theta_{i, w}\left(w^{\prime}\right)=r$.

Theorem 9 Let $w \in W$ be a world of $M$ and $t \in T$ the corresponding state in $\mathscr{T}^{M}$. For any $\varphi$ in $\mathscr{L}_{E P G L}(\boldsymbol{G})$, if $M, w \vDash \varphi$ then $\mathscr{T}^{M}, t \vDash \varphi$.

Proof By induction on the structure of $\varphi$.

We show only the case $\varphi=P_{i}^{r} \psi$ since the others are already covered in Sect. 3. Suppose $M, w \vDash P_{i}^{r} \psi$. Then, by definition $\sum_{w^{\prime} \in \sim_{i}(w): M, w^{\prime} \vDash \varphi} \theta_{i, w}\left(w^{\prime}\right) \geq r$. By inductive hypothesis, $\sum_{t_{-i}^{\prime}: \mathscr{T}, t_{i}^{\prime} \vDash \varphi} \beta_{i}\left(t_{i}\right)\left(t_{-i}^{\prime}\right) \geq r$, where $t_{i}=t_{i}^{\prime}$. Hence $\mathscr{T}^{M}, t \vDash P_{i}^{r} \psi$.

The following corollary then states that the class of type spaces $\mathbf{T}$ and the class of epistemic-probabilistic game models EPGM are equivalent semantics with respect to the language $\mathscr{L}_{E P G L}(\mathbf{G})$.

Corollary 3 A formula $\varphi$ of $\mathscr{L}_{E P G L}(\boldsymbol{G})$ is satisfiable in $\boldsymbol{T}$ iff it is satisfiable in $\mathbf{E P G M}$.

\section{Conclusion}

This work aimed at explicitly showing the formal relations between the two main structures that are used in the literature on epistemic game theory: type spaces and Kripke models. We started with a language for belief and we proceeded by extending the language taken into account. We noticed that with respect to belief the relation can be carried through in a straightforward way, using a rather standard KD45-concept of belief. When we tried to establish a correspondence with respect to a language with both belief and knowledge the situation turned out to be more complicated. Interpreting a S5-concept of knowledge on type spaces in the obvious way gave rise to some interesting properties (as shown in Sect. 3.5) that the usual S5-knowledge operator does not have. This means that Kripke models are less demanding towards the interpretation of knowledge and more suitable to express both knowledge and belief at the same time. The kind of knowledge representable in Kripke models is broader and more general than the interpretation of knowledge in type spaces, whereas the belief representation is basically the same in both structures.

An important thing to stress is that we axiomatized a qualitative logic for type spaces. Since Kripke models are normally qualitative structures, we decided to establish a correspondence from a qualitative point of view in the first place. We then showed what are the qualitative logics (for belief and for both belief and knowledge) and the classes of qualitative Kripke models (i.e., doxastic game models and epistemic game models respectively) corresponding to type spaces (Sects. 2 and 3). We presented here the results for a qualitative language with at most two different operators, $B_{i}$ and $K_{i}$. Using the same line of reasoning we can aim at proving the result for other epistemic attitudes, like strong belief or defeasible knowledge (Baltag and Smets 2008). What we need to do is to enrich the semantics on the side of Kripke models by means of plausibility orders or rankings over worlds instead of simple belief sets (see Baltag and Smets 2008; Spohn 2012). By a further enrichment of Kripke models towards more fine-grained semantics we finally introduced probabilistic Kripke models and we extended the correspondence result to the epistemic-probabilistic language $\mathscr{L}_{E P G L}(\mathbf{G})$ (Sect. 6). 
Concerning the relationship with STIT logic explored in Sect. 5, a further issue we plan to study is the relationship between the logic EDGL extended by distributed knowledge and atemporal group STIT in which agency operators are generalized to groups of agents. As shown by Herzig and Schwarzentruber (2008), the latter is nothing but the product logic S5 ${ }^{n}$ (Gabbay et al. 2003).

Acknowledgments The research of Paolo Galeazzi leading to these results has received funding from the European Research Council under the European Community's Seventh Framework Programme (FP7/20072013)/ERC Grant agreement No. 283963. The authors are also grateful to Sintelnet Project for funding this work.

Open Access This article is distributed under the terms of the Creative Commons Attribution 4.0 International License (http://creativecommons.org/licenses/by/4.0/), which permits unrestricted use, distribution, and reproduction in any medium, provided you give appropriate credit to the original author(s) and the source, provide a link to the Creative Commons license, and indicate if changes were made.

\section{References}

Aumann, R. (1995). Interactive epistemology, Discussion Paper No. 67. Center for Rationality and Interactive Decision Theory. The Hebrew University of Jerusalem.

Aumann, R., \& Brandenburger, A. (1995). Epistemic conditions for nash equilibrium. Econometrica, 63, $1161-1180$.

Balbiani, P., Herzig, A., \& Troquard, N. (2008). Alternative axiomatics and complexity of deliberative stit theories. Journal of Philosophical Logic, 37(4), 387-406.

Baltag, A., \& Smets, S. (2008). A qualitative theory of dynamic interactive belief revision. Texts in Logic and Games, 3, 9-58.

Baltag, A., Smets, S., \& Zvesper, J. (2009). Keep 'Hoping' for rationality: A solution to the backward induction paradox. Synthese, 169, 301-333.

Battigalli, P., \& Bonanno, G. (1999). Recent results on belief, knowledge and the epistemic foundations of game theory. Reasearch in Economics, 53(2), 149-225.

Battigalli, P., \& Siniscalchi, M. (2002). Strong belief and forward induction reasonong. Journal of Economic Theory, 106, 356-391.

Belnap, N., Perloff, M., \& Xu, M. (2001). Facing the future: agents and choices in our indeterminist world. Oxford: Oxford University Press.

Blackburn, P., de Rijke, M., \& Venema, Y. (2001). Modal logic. Cambridge: Cambridge University Press.

Brandenburger, A. (2008). Epistemic game theory: an overview. In S. Durlauf \& L. Blume (Eds.), The new palgrave dictionary of economics. London: Palgrave MacMillan.

Brandenburger, A., \& Dekel, E. (1993). Hierarchies of beliefs and common knowledge. Journal of Economic Theory, 59, 189-198.

Conradie, W., Goranko, V., \& Vakarelov, D. (2006). Algorithmic correspondence and completeness in modal logic i: The core algorithm sqema. Logical Methods in Computer Science, 2(1), 1-26.

Fagin, R. (1994). A quantitative analysis of modal logic. Journal of Symbolic Logic, 59(1), 209-252.

Fagin, R., Geanakoplos, J., Halpern, J., \& Vardi, M. (1999). The hierarchical approach to modeling knowledge and common knowledge. International Journal of Game Theory, 28(3), 331-365.

Fagin, R., Halpern, J., Moses, Y., \& Vardi, M. (1995). Reasoning about Knowledge. Cambridge: MIT Press.

Fagin, R., Halpern, J. Y., \& Vardi, M. Y. (1991). A model-theoretic analysis of knowledge. Journal of the ACM, 91(2), 382-428.

Gabbay, D.M., Kurucz, A., Wolter, F., \& Zakharyaschev, M. (2003). Many-dimensional modal logics: theory and applications. In S. Abramsky, S. Artemov, D. M. Gabbay, A. Kechris, A. Pillay, \& R. A. Shore (Eds.), Studies in Logic and the Foundations of Mathematics (Vol. 148). Amsterdam: Elsevier.

Harsanyi, J. (1967-1968). Games with incomplete informations played by 'bayesian' players. Management Science, 14, 159-182.

Heifetz, A., \& Mongin, P. (2001). Probability logic for type spaces. Games and Economic Behavior, 35, $31-53$. 
Heifetz, A., \& Samet, D. (1998). Knowledge spaces with arbitrarily high rank. Games and Economic Behavior, 22, 260-273.

Herzig, A., \& Schwarzentruber, F. (2008). Properties of logics of individual and group agency. Advances in modal logic, 7, 133-149.

Herzig, A., \& Schwarzentruber, F. (2008). Properties of logics of individual and group agency (regular paper). In: Areces, C., Goldblatt, R. (eds.) Proceedings of advances in modal logic (AiML), College Publications (pp. 133-149).

Horty, J. F. (2001). Agency and deontic logic. Oxford: Oxford University Press.

Klein, D., \& Pacuit, E. (2014). Changing types: Information dynamics for qualitative type spaces. Studia Logica, 102, 297-319.

Kraus, S., \& Lehmann, D. J. (1988). Knowledge, belief and time. Theoretical Computer Science, 58, 155174.

Lorini, E. (2013). On the epistemic foundation for iterated weak dominance: An analysis in a logic of individual and collective attitudes. Journal of Philosophical Logic, 42(6), 863-904.

Lorini, E., \& Schwarzentruber, F. (2010). A modal logic of epistemic games. Games, 1(4), 478-526.

Lorini, E., \& Schwarzentruber, F. (2011). A logic for reasoning about counterfactual emotions. Artificial Intelligence, 175(3-4), 814-847.

Mertens, J. F., \& Zamir, S. (1985). Formulation of Bayesian analysis for games with incomplete information. International Journal of Game Theory, 14, 1-29.

Sahlqvist, H. (1975). Completeness and correspondence in the first and second order semantics for modal logics. In: Proceedings 3rd Scandinavian Logic Symposium 1973, Vol. No. 82 in Studies in Logic.

Spohn, W. (2012). The laws of belief. ranking theory and its philosophical applications. Oxford: Oxford University Press.

Zvesper, J. (2010). Playing with Information. Ph.D. Thesis, University of Amsterdam. 